\title{
Micromechanical modelling of the overall response of plain woven polymer matrix
} composites

\author{
Y. H. Chen and M. H. Aliabadi \\ Department of Aeronautics, Imperial College London, South Kensington Campus, London SW7 2AZ
}

\begin{abstract}
This paper presents a novel approach to micromechanical modelling of woven polymer matrix composite architecture and predicting its overall response including the nonlinear and rate-dependent behaviour. The overall response of plain woven composites is evaluated by introducing a viscoplastic model. The damage evolution of the yarn material and deformation of the woven fabric are investigated by considering a Weibull distribution and shear-modulus discount based formulations, respectively. The explicit meshfree method with time-dependent periodic boundary conditions for UC models that describe the internal architecture of plain woven composites is presented for the first time. The EP121-C15-53 plain woven composite under in-plane normal and off-axis tensile loading conditions, is tested and results are compared to the calculated responses of the composite subjected to tension in the warp direction $\left(0^{\circ}\right)$ at three different strain rates, i.e. $10^{-1} \mathrm{~s}^{-1}, 10^{-3} \mathrm{~s}^{-1}$ and $10^{-5}$ $\mathrm{s}^{-1}$, off-axis direction $\left(45^{\circ}\right)$ at the same three strain rates.
\end{abstract}

Keywords: Plain Woven Composites; Predictive Modelling; Meshfree Methods; Homogenisation; Damage Mechanics

\section{Introduction}

Textile composites are known for their unique combination of light weight and flexibility and their ability to offer a combination of strength and toughness. A wide range of composites structural performances may be tailored with a large family of high-performance fibers assemblies to meet specific requirements. Compared to their unidirectional (UD) counterparts, woven composites have received increased attention, due to their superior mechanical properties, such as improved impact resistance and lower 
notched sensitivity. The attractive properties of woven composites have naturally stimulated extensive studies over the past several decades on predicting the effective elastic properties of these materials, featuring the use of the unit cell (UC) modelling methodology. In such a methodology, a UC model describing the internal architecture of a woven composite under investigation is first developed. Then, constitutive modelling is performed to characterise the microscopic behaviour of the constituents. It is followed by calculating the microscopic stresses and strains of the constituents under prescribed boundary conditions. Finally, by volume-averaging the microscopic quantities and considering the relations between these quantities, the effective properties of the woven composite are predicted.

Depending on the level of sophistication in modelling the internal architecture of woven composites, the UC modelling methodology can be implemented by using either analytical or finite element (FE)-based approaches. In the former case, the internal architecture of woven composites is often discretised as an assemblage of simple blocks in parallel and/or series manners, and, based on such a discretisation, the classical laminate theory (CLT) is often employed to calculate the effective elastic properties (Ishikawa \& Chou, 1982; Shembekar \& Naik, 1992). Clearly, the simplifications of geometrical features, which are necessary to implement the analytical nature, make analytical approaches computationally efficient. However, they may unavoidably limit the accuracy of predicted results due to the analytical nature.

The construction of representative volume element (RVEs) for textile architecture composite is rather complicated due to the complex geometry. Several commercial and open source software tools are available to help with this task such as (Wentorf, Collar, Shephard \& Fish, 1999, WiseTex: Lomov, Gusakov, Huysmans, Prodromou \& Verpoest, 2000) and TexGen University of Nottingham, 2019). For review of homogenisation approaches for textile composites, readers should consult Fish and Shek (2000) and Aliabadi (2015). 
Several meso-scale models considering the internal features of woven composites (such as the cross-sectional shape and waviness of yarns) have been developed for accurate prediction of the effective properties (Bacarreza, Abe, Aliabadi \& Ragavan, 2012; Bogdanovich, 2006; Whitcomb \& Tang, 2001). Durville (2010) investigated the contactfriction interactions occurring within the collection of fibers. Hallett, Green, and EI Said (2015) presented a multi-scale approach to kinematic modelling 3D woven fabric deformations and defects. Lomov \& Verpoest (2000) presented a model to account for the compression of yarns and the change of yarn crimp under pressure. Wen and Aliabadi (2009) proposed a meshfree-based UC modelling approach for predicting the elastic properties of woven composites. This approach features developing a geometry model that describes the internal features of woven composites using sophisticated functions and implementing the geometry model. Later, Li, Wen and Aliabadi (2011) extend the formulation to predict the effective elastic properties of 3D woven composites. The studies by Wen et al. (2009) and Li et al. (2011) were based on direct boundary conditions for simplicity and thus did not necessarily satisfy the Hill-Mandel principle (Hill, 1965). Given that the Hill-Mandel principle is a prior condition for the homogenised properties accurately representing the macroscopic properties of the material under investigation, one of the objectives of this research was to develop a meshfree-based UC modelling approach capable of implementing periodic boundary conditions (PBCs), which always satisfy the Hill-Mandel principle. Also, the existing UC modelling approaches mainly focus on predicting the effective elastic properties, limiting the possibility of understanding woven composites beyond the static, elastic regime. Therefore, another objective UC modelling approach in this paper is to further to the extent of predicting the overall response of woven composites including the nonlinear, rate-dependent behaviour under dynamic loading conditions. 


\section{Unit cell modelling}

\subsection{UC Identification and general assumptions}

This research is based on the most fundamental type of woven composites, i.e. plain woven composites with simple stacking, see Fig. 1(a). Here, the matrix phase is hidden to reveal the internal fibre yarns. To reduce the complexity in geometry modelling and the computational cost of numerical analysis, the minimum unit cell (mUC), which is derived by exploiting all symmetries in the composites, is utilised. Prior to modelling the internal features, some specimens based on the plain woven composite under investigation were fabricated to observe the cross-sectional shape and waviness of fibres yarns, Figs. 2(a) and 2(b). Based on these micrographs and the curve fittings shown in Figs. 2(c) and 2(d), it is assumed that both the cross-section and waviness of a general warp or fill yarn can be described using cosine functions. Also, each yarn is assumed to have a constant crosssectional shape throughout the whole yarn, as indicated by the yarns highlighted using the dashed curves in Figs. 2(a) and 2(b). Further, the overall thickness of a fibre yarn is assumed to be half of the fabric thickness given that there are only two fibre yarns in the through-the-thickness direction in a layer of woven fabric.

\subsection{Normal unit cell}

Fig. 1(b) shows the normal mUC of plain woven composites. Here, the term "normal" is used to suggest that this mUC is based on an orthogonal coordinate system with its $\mathrm{X}, \mathrm{Y}$ and $\mathrm{Z}$ axes parallel to the warp, fill and through-the-thickness directions, respectively, as opposed to the "off-axis" mUC, which will be shown later. The normal mUC is used for the homogenisation in the warp/fill directions (i.e. $0^{\circ} / 90^{\circ}$ ), while the off-axis $\mathrm{mUC}$ is for that in the off-axis directions (e.g. $45^{\circ}$ ). In the normal mUC, parameter $L$ is used to represent the edge length of the mUC. In addition, a dimensionless parameter, $\lambda$, which is defined as the ratio of the half-width of the warp or fill yarn $(\lambda L)$ to the edge length $(L)$, is introduced to accommodate plain woven composites with either a tight or loose fabric. 
Based on the assumptions discussed above and the geometrical configurations illustrated in Fig. 1(b), the lower and upper surfaces of the warp and weft yarns, as well as their undulation angles (i.e. waviness) with respect to plane XOY, can be analytically described as follows, where subscripts "lo" and "up" refer to the lower and upper surfaces of the yarn:

$$
\begin{gathered}
z_{\text {warp }}^{\text {lo/up }}=\frac{H}{4}\left(2+\cos \frac{\pi x}{2 L} \mp \cos \frac{\pi y}{2 \lambda L}\right) \\
z_{\text {fill }}^{\text {lo/up }}=\frac{H}{4}\left(2 \mp \cos \frac{\pi x}{2 \lambda L}-\cos \frac{\pi y}{2 L}\right) \\
\theta_{\text {warp/fill }}=\tan ^{-1}\left(\mp \frac{\pi H}{8 L} \sin \frac{\pi x}{2 L}\right)
\end{gathered}
$$

\subsection{Off-axis unit cell}

Fig. 3 shows the off-axis mUC of plain woven composites, which can also be derived after performing a domain reduction procedure similar to the one shown in Fig. 1(a). As indicated in Fig. 3, it is problematic to describe the fibre yarns directly in the local coordinate system (LCS) of the off-axis mUC, i.e. OXYZ. A simpler way is to initially describe the fibre yarns in the global coordinate system (GCS), i.e. $\mathrm{O}^{\prime} \mathrm{X}^{\prime} \mathrm{Y}^{\prime} \mathrm{Z}^{\prime}$, and then to transform the resulting equations back into the LCS. Based on the two-step procedure, the equations for describing the internal features of the off-axis mUC can be obtained, as follows:

$$
\begin{gathered}
Z_{\mathrm{warp}}^{\mathrm{lo} / \mathrm{up}}=\frac{H}{4}\left(2+\cos \frac{\sqrt{2} \pi(x+y)}{4 L} \mp \cos \frac{\sqrt{2} \pi(x-y)}{4 \lambda L}\right) \\
Z_{\mathrm{fill} 1}^{\mathrm{lo} / \mathrm{up}}=\frac{H}{4}\left(2 \mp \cos \frac{\sqrt{2} \pi(x+y)}{4 \lambda L}-\cos \frac{\sqrt{2} \pi(x-y)}{4 L}\right) \\
Z_{\text {fill } 2}^{\mathrm{lo} / \mathrm{up}}=\frac{H}{4}\left(2 \pm \cos \left(\frac{\sqrt{2} \pi(x+y)}{4 \lambda L}-\frac{\pi}{\lambda}\right)+\cos \frac{\sqrt{2} \pi(x-y)}{4 L}\right) \\
\theta_{\text {warp }}=\tan ^{-1}\left(-\frac{\pi H}{8 L} \sin \frac{\sqrt{2} \pi(x+y)}{4 L}\right) \\
\varphi_{\text {fill1 } / \text { fill2 }}=\tan ^{-1}\left(\mp \frac{\pi H}{8 L} \sin \frac{\sqrt{2} \pi(x-y)}{4 L}\right)
\end{gathered}
$$

\section{Constitutive modelling}

Predicting the overall response of woven composites under complex loading conditions is a challenging task. Thus, the present work focuses on the response under in-plane tensile and 
shear loading conditions, as a start point. Also, the constitutive modelling of fibre yarns will be described in its LCS for simplicity, given the fact that the material orientations of fibre yarns have been defined in Section 2 and that their locally defined constitutive relations can be readily transformed back to the GCS of the unit cell (Li et al., 2011), as follows:

$$
\begin{gathered}
\boldsymbol{\sigma}^{g}=\mathbf{T}^{\mathrm{T}} \boldsymbol{\sigma}^{l} \\
\boldsymbol{\varepsilon}^{g}=\left(\mathbf{T}^{\mathrm{T}}\right)^{-1} \boldsymbol{\varepsilon}^{l} \\
\mathbf{C}^{g}=\mathbf{T C}^{l} \mathbf{T}^{\mathrm{T}}
\end{gathered}
$$

where superscripts $g$ and $l$ denote that the quantities are based in the GCS and LCS, respectively, and $\mathbf{T}$ is a transformation matrix defined in Li et al. (2011, p. 4).

\subsection{Material description}

The plain woven composite under investigation is based on the EP121-C15-53 prepreg (Gurit, 2019). This prepreg is made of 3k HTA40 carbon fibres pre-impregnated with a 53\% EP121 resin matrix, which is a toughened, self-extinguishing epoxy resin. This composite has an overall fibre volume fraction (FVF) of $38.7 \%$ and an intra-yarn FVF of 67.5\%. For simplicity, this material system will be termed as EP121-C15-53.

\subsection{Constitutive modelling of polymer matrix}

\subsubsection{Isotropic elasticity of polymer matrix}

The epoxy resin used this research, which is a typical polymer, also features linear elastic behaviour at small strains and become nonlinear and rate-dependent if the strain applied exceeds the linearity threshold. In the linear elastic regime, the constitutive behaviour of the epoxy resin can be described using Hooke's law, with its elasticity matrix being defined by:

$$
\mathbf{C}_{\mathrm{m}}=\left[\begin{array}{cccccc}
\frac{E_{\mathrm{m}}\left(1-v_{\mathrm{m}}\right)}{\Delta_{\mathrm{m}}} & \frac{E_{\mathrm{m}} v_{\mathrm{m}}}{\Delta_{\mathrm{m}}} & \frac{E_{\mathrm{m}} v_{\mathrm{m}}}{\Delta_{\mathrm{m}}} & 0 & 0 & 0 \\
\frac{E_{\mathrm{m}} v_{\mathrm{m}}}{\Delta_{\mathrm{m}}} & \frac{E_{\mathrm{m}}\left(1-v_{\mathrm{m}}\right)}{\Delta_{\mathrm{m}}} & \frac{E_{\mathrm{m}} v_{\mathrm{m}}}{\Delta_{\mathrm{m}}} & 0 & 0 & 0 \\
\frac{E_{\mathrm{m}} v_{\mathrm{m}}}{\Delta_{\mathrm{m}}} & \frac{E_{\mathrm{m}} v_{\mathrm{m}}}{\Delta_{\mathrm{m}}} & \frac{E_{\mathrm{m}}\left(1-v_{\mathrm{m}}\right)}{\Delta_{\mathrm{m}}} & 0 & 0 & 0 \\
0 & 0 & 0 & G_{\mathrm{m}} & 0 & 0 \\
0 & 0 & 0 & 0 & G_{\mathrm{m}} & 0 \\
0 & 0 & 0 & 0 & 0 & G_{\mathrm{m}}
\end{array}\right]
$$


where $E_{\mathrm{m}}, v_{\mathrm{m}}$ and $G_{\mathrm{m}}$ are Young's modulus, Poison's ratio and the shear modulus, and $\Delta_{\mathrm{m}}$ is defined by:

$$
\Delta_{\mathrm{m}}=\left(1+v_{\mathrm{m}}\right)\left(1-2 v_{\mathrm{m}}\right)
$$

\subsubsection{Nonlinearity and rate-dependence of polymer matrix}

The viscoplasticity model developed by Goldberg and Stouffer (1998) is employed to characterise the nonlinear, rate-dependent behaviour of the polymer matrix. It describes the deformation of a polymer in a rate form, with the inelastic strain rate $\left(\dot{\varepsilon}_{i j}^{\mathrm{I}}\right)$ being modelled as a function of a number of material parameters and a tensorial state variable, which in return evolves with the inelastic strain rate, as follows:

$$
\begin{gathered}
\dot{\varepsilon}_{i j}^{\mathrm{I}}=D_{0} \exp \left(-\frac{1}{2}\left(\frac{Z_{0}^{2}}{3 K_{2}}\right)^{n_{\mathrm{r}}}\right) \frac{S_{i j}-\Omega_{i j}}{\sqrt{K_{2}}} \\
\dot{\Omega}_{i j}=\frac{2}{3} q \Omega_{\mathrm{m}} \dot{\varepsilon}_{i j}^{\mathrm{I}}-q \Omega_{i j} \dot{\varepsilon}_{\mathrm{eff}}^{\mathrm{I}} \\
\dot{\varepsilon}_{\mathrm{e}}^{\mathrm{I}}=\sqrt{\frac{2}{3} \dot{\varepsilon}_{i j}^{\mathrm{I}} \dot{\varepsilon}_{i j}^{\mathrm{I}}}
\end{gathered}
$$

In the above equations, $D_{0}, Z_{0}, n_{\mathrm{r}}, q$ and $\Omega_{\mathrm{m}}$ are material parameters representing the maximum inelastic strain rate, the initial hardness, the rate-dependent coefficient, the hardening rate and the stress at saturation, respectively. $S_{i j}$ refers to the deviatoric stress. The state variable, $\Omega_{i j}$, is termed as the internal stress and used to model the resistance to molecular flow. $K_{2}$ is the second invariant of the overstress, i.e. $S_{i j}-\Omega_{i j} . \dot{\varepsilon}_{\text {eff }}^{\mathrm{I}}$ stands for the effective inelastic strain rate.

It should be noted the variables defined in Eqs. (12) (14) evolve with respect to each other, resulting in a set of constitutive equations without closed solutions. As suggested by Tabiei and Ivanov (2007), a reasonable approach is to employ the four-step Runge-Kutta method in combination with the explicit central difference method to incrementally solve these equations. For the determination of the material constants, $D_{0}$ is recommended to take $10^{4}$ times the maximum strain rate applied (Goldberg et al., 1998), $\Omega_{\mathrm{m}}$ is often estimated to 
be $50 \% \sim 75 \%$ of the maximum uniaxial stress at saturation, and $q$ is assumed to be the solution of Eq. (15), where $\varepsilon_{\mathrm{s}}^{\mathrm{I}}$ is the inelastic strain at saturation:

$$
\exp \left(-q \varepsilon_{\mathrm{s}}^{\mathrm{I}}\right)=0.01
$$

To determine the remaining parameters, mathematical operations on the uniaxial form of the viscoplasticity model should be conducted to obtain the following relation (Goldberg et al., 1998):

$$
y=-2 n_{\mathrm{r}} x+2 n_{\mathrm{r}} \ln \left(Z_{0}\right)
$$

where

$$
\begin{gathered}
x=\ln \left(\sigma_{\mathrm{s}}-\Omega_{\mathrm{m}}\right) \\
y=\ln \left[-2 \ln \left(\frac{\sqrt{3} \dot{\varepsilon}_{0}}{2 D_{0}}\right)\right]
\end{gathered}
$$

From Eq. (16), it is evident that $y$ correlates linearly with $x$. Therefore, a set of uniaxial tensile tests can be conducted at different constant strain rates such that a group of data points $\left(x_{i}, y_{i}\right)$ can be collected to perform a least-squares regression analysis. By using the results from the regression analysis, the value of $n_{\mathrm{r}}$ and $Z_{0}$ can be fitted.

\subsubsection{Damage initiation and evolution of polymer matrix}

As the Goldberg model discussed above describes only the deformation of a polymer prior to failure, suitable functions must be defined to characterise the post-failure behaviour.

Considering that polymers exhibit a low degree of sensitivity to stress after saturation, the maximum strain criterion is employed to identify the point where damage initiates. Thus, the failure function of the polymer matrix is defined by:

$$
f_{\mathrm{m}}=\left(\frac{\varepsilon_{\mathrm{eff}}}{\varepsilon_{\mathrm{mf}}}\right)^{2}=1
$$

where $\varepsilon_{\text {eff }}$ and $\varepsilon_{\mathrm{mf}}$ refer to the effective strain and failure strain, respectively. It is worth noting that the value of the failure strain may vary with strain rate. To take this effect into account, the logarithm formulation that was originally proposed by Weeks and Sun (1998) for describing the rate-dependence of composites is adapted to scale the failure strain, as follows: 


$$
\varepsilon_{\mathrm{mf}}=\left\{\begin{array}{cl}
\varepsilon_{\mathrm{m} 0}\left(1 \pm C_{\mathrm{m} \varepsilon} \ln \left|\frac{\dot{\varepsilon}}{\dot{\varepsilon}_{0}}\right|\right) & |\dot{\varepsilon}|>\dot{\varepsilon}_{0} \\
\varepsilon_{\mathrm{m} 0} & |\dot{\varepsilon}| \leq \dot{\varepsilon}_{0}
\end{array}\right.
$$

where $\dot{\varepsilon}_{0}$ refers to the reference strain rate, $\varepsilon_{\mathrm{m} 0}$ is the failure strain measured at the reference strain rate, and $C_{\mathrm{m} \varepsilon}$ is a parameter used to scale the failure strain and can be determined by performing a least-squares regression analysis on a group of uniaxial tensile tests conducted at different constant strain rates. In the above equation, the positive (or negative) sign should be used if the failure strain is found to increase (decrease) with strain rate.

In terms of the damage evolution of the polymer matrix, it is assumed to follow a softening law similar to that for describing the damage evolution of the yarn material, which will be addressed later. This law assumes that damage develops following a formulation based on Weibull distribution (Weibull, 1939), as follows:

$$
\omega_{\mathrm{m}}=\left\{\begin{array}{cl}
0 & \left|\frac{\varepsilon_{\mathrm{eff}}}{\varepsilon_{\mathrm{mf}}}\right| \leq 1 \\
1-\exp \left[-\frac{1}{\exp (1)}\left(\left(\left|\frac{\varepsilon_{\mathrm{eff}}}{\varepsilon_{\mathrm{mf}}}\right|\right)^{\beta_{\mathrm{m}}}-1\right)-\right] & \left|\frac{\varepsilon_{\mathrm{eff}}}{\varepsilon_{\mathrm{mf}}}\right|>1
\end{array}\right.
$$

where $\beta_{\mathrm{m}}$ is a constant used to describe the rate of damage evolution. Based on Eq. (21), the degradation of material integrity due to damage can be described by modifying the elasticity matrix defined in Eq. (10) into $C_{m}\left(1-\omega_{m}\right)$.

\subsection{Constitutive modelling of yarn material}

\subsubsection{Transversely isotropic elasticity of yarn material}

As fibre yarns in woven composites are essentially unidirectional (UD) composites in the LCS, they can be assumed to be transversely isotropic with linearly elastic response prior to failure, followed by anisotropic post-failure behaviour. Thus, the elasticity matrix of the yarn material is defined by: 


$$
\mathbf{C}_{\mathrm{y}}=\left[\begin{array}{cccccc}
\frac{E_{\mathrm{L}}\left(1-v_{\mathrm{TT}}\right)}{\Delta} & \frac{E_{\mathrm{T}} v_{\mathrm{LT}}}{\Delta} & \frac{E_{\mathrm{T}} v_{\mathrm{LT}}}{\Delta} & 0 & 0 & 0 \\
\frac{E_{\mathrm{L}} v_{\mathrm{TL}}}{\Delta} & \frac{E_{\mathrm{T}}\left(1-v_{\mathrm{LT}} v_{\mathrm{TL}}\right)}{\Delta\left(1+v_{\mathrm{TT}}\right)} & \frac{E_{\mathrm{T}}\left(v_{\mathrm{TT}}+v_{\mathrm{LT}} v_{\mathrm{TL}}\right)}{\Delta\left(1+v_{\mathrm{TT}}\right)} & 0 & 0 & 0 \\
\frac{E_{\mathrm{L}} v_{\mathrm{TL}}}{\Delta} & \frac{E_{\mathrm{T}}\left(v_{\mathrm{TT}}+v_{\mathrm{LT}} v_{\mathrm{TL}}\right)}{\Delta\left(1+v_{\mathrm{TT}}\right)} & \frac{E_{\mathrm{T}}\left(1-v_{\mathrm{LT}} v_{\mathrm{TL}}\right)}{\Delta\left(1+v_{\mathrm{TT}}\right)} & 0 & 0 & 0 \\
0 & 0 & 0 & G_{\mathrm{LT}} & 0 & 0 \\
0 & 0 & 0 & 0 & G_{\mathrm{TT}} & 0 \\
0 & 0 & 0 & 0 & 0 & G_{\mathrm{TL}}
\end{array}\right]
$$

where $E_{\mathrm{L}}$ and $E_{\mathrm{T}}$ are the elastic moduli in the longitudinal and transverse directions, $v_{i j}(i, j=\mathrm{L}, \mathrm{T})$ is Poisson's ratio relating to a contraction in direction $j$ caused by a tension in direction $i, G_{\mathrm{LT}}, G_{\mathrm{TT}}$ and $G_{\mathrm{TL}}$ are the shear moduli, and $\Delta$ is defined by:

$$
\Delta=1-v_{\mathrm{TT}}-2 v_{\mathrm{LT}} v_{\mathrm{TL}}
$$

\subsubsection{Failure criteria for yarn material}

Given that the yarn material is locally treated as a UD composite material, its failure can be identified using the criteria developed for UD composites. In this research, Hashin's failure criteria (Hashin, 1980) were generalised to identify the damage initiation of the yarn material, in view of their physically based nature and reasonable capability of predicting composite failure (Kress, 2012; Xu, Yang, Guan \& Cantwell, 2016). Six functions were defined to describe six failure modes, as follows:

1) $f_{1 \mathrm{fT}}\left(\sigma_{11} \geq 0\right)$ - Fibre failure under tension is assumed to be as a result of a quadratic interaction between the tensile and shear stresses:

$$
f_{1 \mathrm{fT}}=\left(\frac{\left\langle\sigma_{11}\right\rangle}{X_{\mathrm{T}}}\right)^{2}+\frac{\tau_{12}^{2}}{S_{\mathrm{LT}}^{2}}+\frac{\tau_{31}^{2}}{S_{\mathrm{TL}}^{2}}=1
$$

2) $f_{1 \mathrm{fC}}\left(\sigma_{11}<0\right)$ - Fibre failure under compression is defined based on the normal compressive stresses in the longitudinal and transverse directions:

$$
f_{1 \mathrm{fC}}=\left(\frac{-\sigma_{11}-\left\langle-\frac{\sigma_{22}+\sigma_{33}}{2}\right)}{X_{\mathrm{C}}}\right)^{2}=1
$$

3) $f_{2 \mathrm{mC}}\left(\sigma_{22} \geq 0\right)$ - Matrix cracking under in-plane transverse tension is treated as a result of a quadratic interaction between the transverse tensile and shear stresses: 


$$
f_{2 \mathrm{mC}}=\left(\frac{\left\langle\sigma_{22}\right\rangle}{Y_{\mathrm{T}}}\right)^{2}+\frac{\tau_{12}^{2}}{S_{\mathrm{LT}}^{2}}+\frac{\tau_{23}^{2}}{S_{\mathrm{TT}}^{2}}=1
$$

4) $f_{2 \mathrm{~ms}}\left(\sigma_{22}<0\right)$ - Matrix shear failure under in-plane transverse compression is defined directly using the criterion proposed by Hashin (1980):

$$
f_{2 \mathrm{mS}}=\left(\frac{\left\langle-\sigma_{22}\right)}{2 S_{\mathrm{TT}}}\right)^{2}+\left[\left(\frac{Y_{\mathrm{C}}}{2 S_{\mathrm{TT}}}\right)^{2}-1\right] \frac{\sigma_{22}}{Y_{\mathrm{C}}}+\frac{\tau_{12}^{2}}{S_{\mathrm{LT}}^{2}}=1
$$

5) $f_{3 \mathrm{mC}}\left(\sigma_{33} \geq 0\right)$ - Matrix cracking under out-of-plane transverse tension is formulated in a manner similar to failure mode 3:

$$
f_{3 \mathrm{mC}}=\left(\frac{\left\langle\sigma_{33}\right\rangle}{z_{\mathrm{T}}}\right)^{2}+\frac{\tau_{23}^{2}}{S_{\mathrm{TT}}^{2}}+\frac{\tau_{31}^{2}}{S_{\mathrm{TL}}^{2}}=1
$$

6) $f_{3 \mathrm{~ms}}\left(\sigma_{33}<0\right)$ - Matrix shear failure under out-of-plane transverse compression is defined similarly to failure mode 4 :

$$
f_{3 \mathrm{mS}}=\left(\frac{\left(-\sigma_{33}\right)}{2 S_{\mathrm{TT}}}\right)^{2}+\left[\left(\frac{Z_{\mathrm{C}}}{2 S_{\mathrm{TT}}}\right)^{2}-1\right] \frac{\sigma_{33}}{Z_{\mathrm{C}}}+\frac{\tau_{31}^{2}}{S_{\mathrm{TL}}^{2}}=1
$$

In the above failure functions, $\sigma_{i i}$ and $\tau_{i j}$ denotes the normal and shear stress components, $X_{\mathrm{T}}$ and $X_{\mathrm{C}}$ represent the tensile and compressive strengths in the fibre direction, $Y_{\mathrm{T}}$ and $Y_{\mathrm{C}}$ refers to the tensile and compressive strengths in the in-plane transverse direction, $Z_{\mathrm{T}}$ and $Z_{\mathrm{C}}$ are the tensile and compressive strengths in the out-of-plane transverse direction, $S_{\mathrm{LT}}$, $S_{\mathrm{TT}}$ and $S_{\mathrm{TL}}$ are the shear strengths of the yarn material in the corresponding planes, and symbol $\langle\quad\rangle$ denotes the Macaulay brackets.

\subsubsection{Damage evolution of yarn material}

An improved Weibull distribution based formulation is proposed to describe the damage evolution of the yarn material, with its uniaxial form written as follows:

$$
\omega=\left\{\begin{array}{cc}
0 & E|\varepsilon|<X \\
1-\exp \left(-\frac{1}{m_{\mathrm{s}} \exp (1)}\left(\left(\frac{E|\varepsilon|}{\mathrm{X}}\right)^{\beta}-1\right)^{m_{\mathrm{s}}}\right) & E|\varepsilon| \geq X
\end{array}\right.
$$

Here, $E$ refers to the elastic modulus, $\varepsilon$ is the strain, $X$ represents the strength, and $\mathrm{m}_{\mathrm{s}}$ and $\beta$ are two material parameters. It should be noted that the above formulation is proposed to avoid the problem inherently existing in the standard formulation (Aminjikarai \& Tabiei, 2007), where damage accumulates at zero strain and reaches a non-zero value at the point of 
damage initiation. In the improved formulation, parameter $m_{\mathrm{s}}$ is retained to ensure that it still complies with the original Weibull distribution and is set to be unity for simplicity, and $\beta$ is introduced to describe the rate of damage evolution. Based on the proposed formulation, the damage variables corresponding to the six failure modes of the yarn material are defined in the following compact form, where the components in $\boldsymbol{F}_{\mathrm{y}}$ represent the six failure functions defined in Section 3.2.2 and $\beta_{i}(i=1,2, \ldots, 6)$ is the damage evolution coefficient corresponding to the $i$ th failure mode:

$$
\begin{gathered}
\boldsymbol{\omega}_{\mathrm{y}}=\left\{\begin{array}{ccccc}
\boldsymbol{O} & \boldsymbol{F}_{\mathrm{y}}<\mathbf{1} \\
1-\exp \left(-\frac{1}{\exp (1)}\left(\boldsymbol{F}_{\mathrm{y}}{ }^{\boldsymbol{1}}-\mathbf{1}\right)\right) & \boldsymbol{F}_{\mathrm{y}} \geq \mathbf{1}
\end{array}\right. \\
\boldsymbol{\omega}_{\mathrm{y}}=\left\{\begin{array}{llllll}
\omega_{1 \mathrm{fT}} & \omega_{1 \mathrm{fC}} & \omega_{2 \mathrm{mC}} & \omega_{2 \mathrm{mS}} & \omega_{3 \mathrm{mC}} & \omega_{3 \mathrm{~ms}}
\end{array}\right\}^{\mathrm{T}} \\
\boldsymbol{\beta}=\left\{\begin{array}{llllll}
\beta_{1} & \beta_{2} & \beta_{3} & \beta_{4} & \beta_{5} & \beta_{6}
\end{array}\right\}^{\mathrm{T}} \\
\boldsymbol{F}_{\mathrm{y}}=\left\{\begin{array}{llllll}
f_{1 \mathrm{fT}} & f_{1 \mathrm{fC}} & f_{2 \mathrm{mC}} & f_{2 \mathrm{mS}} & f_{3 \mathrm{mC}} & f_{3 \mathrm{~ms}}
\end{array}\right\}^{\mathrm{T}}
\end{gathered}
$$

It is worth noting that the material constants in Eq. (33) can be tuned by conducting uniaxial tension or compression tests. As conducting in-situ tests on fibre yarns to determine these constants is problematic, a practical solution is to use UD composite specimens that have the same constituents and FVF as the yarn material. Based on the experimental data, each constant can be determined by minimising a least-squares function that sums the residuals between the stresses measured at the post-failure stage, $\sigma_{j}(j=1,2, \ldots, n)$ and the predicted stresses, $\hat{\sigma}_{j}$ :

$$
g\left(\beta_{i}\right)=\sqrt{\left(\sigma_{1}-\hat{\sigma}_{1}\right)^{2}+\cdots+\left(\sigma_{j}-\hat{\sigma}_{j}\right)^{2}+\cdots+\left(\sigma_{n}-\hat{\sigma}_{n}\right)^{2}}
$$

where $\hat{\sigma}_{j}$ can be calculated based on the measured post-failure strain, $\varepsilon_{j}$, as follows:

$$
\hat{\sigma}_{j}=\exp \left(-\frac{1}{\exp (1)}\left(\left(\frac{E\left|\varepsilon_{j}\right|}{\mathrm{x}}\right)^{\beta_{i}}-1\right)\right) E \varepsilon_{j}
$$


To account for the degradation of material stiffness due to damage, the elasticity matrix defined in Eq. (22) is modified into a damaged elasticity matrix, $\mathbf{C}(\omega)$, which can be expressed as the inverse of $\mathbf{S}(\omega)$, as follows:

$$
\begin{gathered}
\mathbf{C}(\omega)=\mathbf{S}^{-1}(\omega)=\left[\begin{array}{ccc}
\mathbf{S}_{\mathrm{d}}^{-1}(\omega) & \mathbf{0} \\
\mathbf{0} & \mathbf{S}_{\mathrm{S}}^{-1}(\omega)
\end{array}\right] \\
\mathbf{S}_{\mathrm{d}}(\omega)=\left[\begin{array}{ccc}
\frac{1}{E_{\mathrm{L}}\left(1-\omega_{1}\right)} & -\frac{v_{\mathrm{LT}}}{E_{\mathrm{L}} \sqrt{\left(1-\omega_{1}\right)\left(1-\omega_{2}\right)}} & -\frac{v_{\mathrm{TL}}}{E_{\mathrm{T}} \sqrt{\left(1-\omega_{1}\right)\left(1-\omega_{3}\right)}} \\
-\frac{v_{\mathrm{LT}}}{E_{\mathrm{L}} \sqrt{\left(1-\omega_{1}\right)\left(1-\omega_{2}\right)}} & \frac{1}{E_{\mathrm{T}}\left(1-\omega_{2}\right)} & -\frac{v_{\mathrm{TT}}}{E_{\mathrm{T}} \sqrt{\left(1-\omega_{2}\right)\left(1-\omega_{3}\right)}} \\
-\frac{v_{\mathrm{TL}}}{E_{\mathrm{T}} \sqrt{\left(1-\omega_{1}\right)\left(1-\omega_{3}\right)}} & -\frac{v_{\mathrm{TT}}}{E_{\mathrm{T}} \sqrt{\left(1-\omega_{2}\right)\left(1-\omega_{3}\right)}} & \frac{1}{E_{\mathrm{T}}\left(1-\omega_{3}\right)}
\end{array}\right] \\
\mathbf{S}_{\mathrm{S}}(\omega)=\left[\begin{array}{ccc}
\frac{1}{G_{\mathrm{LT}}\left(1-\omega_{4}\right)} & 0 & 0 \\
0 & \frac{1}{G_{\mathrm{TT}}\left(1-\omega_{5}\right)} & 0 \\
0 & 0 & \frac{1}{G_{\mathrm{TL}}\left(1-\omega_{6}\right)}
\end{array}\right]
\end{gathered}
$$

where

$$
\begin{gathered}
\omega_{1}=\left(\omega_{1 \mathrm{fT}}\left\langle\sigma_{11}\right\rangle-\omega_{1 \mathrm{fC}}\left\langle-\sigma_{11}\right\rangle\right) / \sigma_{11} \\
\omega_{2}=\left(\omega_{2 \mathrm{mC}}\left\langle\sigma_{22}\right\rangle-\omega_{2 \mathrm{~ms}}\left\langle-\sigma_{22}\right\rangle\right) / \sigma_{22} \\
\omega_{3}=\left(\omega_{3 \mathrm{mC}}\left\langle\sigma_{33}\right\rangle-\omega_{3 \mathrm{mS}}\left\langle-\sigma_{33}\right\rangle\right) / \sigma_{33} \\
\omega_{4}=1-\left(1-\omega_{1 \mathrm{fT}}\right)\left(1-\omega_{1 \mathrm{fC}}\right)\left(1-\omega_{2 \mathrm{mC}}\right)\left(1-\omega_{2 \mathrm{mS}}\right) \\
\omega_{5}=1-\left(1-\omega_{2 \mathrm{mC}}\right)\left(1-\omega_{2 \mathrm{mS}}\right)\left(1-\omega_{3 \mathrm{mC}}\right)\left(1-\omega_{3 \mathrm{mS}}\right) \\
\omega_{6}=1-\left(1-\omega_{3 \mathrm{mC}}\right)\left(1-\omega_{3 \mathrm{mS}}\right)\left(1-\omega_{1 \mathrm{fT}}\right)\left(1-\omega_{1 \mathrm{fC}}\right)
\end{gathered}
$$

\subsubsection{Deformation of woven fabric under shear}

Under in-plane tensile loadings in the warp or fill direction, the straightening effects of fibre yarns in plain woven composites is limited, due to a low degree of waviness and the constraints mutually imposed between the warp and fill yarns. However, as a result of the polymer matrix having relatively low stiffness, plain woven composites may be deformed significantly under in-plane shear loadings, in the form of the warp and fill yarns reorienting from the mutually perpendicular state to a fully locked position. In the present research, an approach developed by Tabiei and Ivanov (2002) for describing the deformation of flexible woven fabrics is adapted. Specifically, the in-plane shear modulus $G_{\mathrm{LT}}$ is modified into 
$\mu G_{\mathrm{LT}}$, where $\mu$ is a discount factor used to model the reorientation of woven fabric. This factor is assumed to have a value of $G_{\mathrm{m}} / G_{\mathrm{LT}}$ if the warp and weft yarns are in the original state and linearly increase to unity when the yarns are gradually compacted to a fully locked position. Considering this assumption and the geometrical relations in the fully locked positions, the value of the discount factor at different strain conditions can be determined for the off-axis mUC, as follows, where $G_{\mathrm{m}}$ is the shear modulus of the polymer matrix:

$$
\mu=\left\{\begin{array}{cc}
\frac{\left(G_{\mathrm{LT}}^{2}-G_{\mathrm{m}}\right)\left\langle\varepsilon_{11}\right\rangle+G_{\mathrm{m}} \varepsilon_{\mathrm{a}}}{G_{\mathrm{LT}} \varepsilon_{\mathrm{a}}} & \varepsilon_{\mathrm{a}} \leq\left\langle\varepsilon_{11}\right\rangle<0 \\
\frac{\left(G_{\mathrm{LT}}^{2}-G_{\mathrm{m}}\right)\left\langle\varepsilon_{11}\right\rangle+G_{\mathrm{m}} \varepsilon_{\mathrm{b}}}{G_{\mathrm{LT}} \varepsilon_{\mathrm{b}}} & \varepsilon_{\mathrm{b}}>\left\langle\varepsilon_{11}\right\rangle \geq 0 \\
1 & \text { otherwise }
\end{array}\right.
$$

where $\left\langle\varepsilon_{11}\right\rangle$ is the volume average strain applied in the X-direction of the off-axis mUC, and $\varepsilon_{\mathrm{a}}$ and $\varepsilon_{\mathrm{b}}$ are defined by:

$$
\begin{aligned}
& \varepsilon_{\mathrm{a}}=\sqrt{2} \sin (0.5 \arcsin (\lambda))-1 \\
& \varepsilon_{\mathrm{b}}=\sqrt{2} \cos (0.5 \arcsin (\lambda))-1
\end{aligned}
$$

\section{Meshfree implementation}

\subsection{The element-free Galerkin method}

Meshfree methods are a relatively new class of numerical methods, differing from the finite element method (FEM) in that a meshfree method discretises a problem domain only using arbitrarily distributed nodes, rather than elements, as shown in Fig. 4. The node-based nature makes it possible to implicitly implement the proposed UC models without building the internal geometries, which will be discussed later. Another difference is that a meshfree method approximates unknown field variables (e.g. displacements) based on support domains, which may have different shapes and overlaps. In this research, the element-free Galerkin (EFG) method (Belytschko, Lu \& Gu, 1994) is employed for the numerical implementation. Here, it should be noted that instead of using the moving least squares (MLS) method, the moving kriging (MK) technique $(\mathrm{Gu}, 2002)$ is adopted to construct meshfree shape functions to address the problem of the MLS method being unable to 
enforce essential boundary conditions exactly due to the lack of the Kronecker Delta function property.

The homogenisation of the response of plain woven composites is essentially a dynamic problem with initial and boundary conditions. By applying the EFG method, the discretised system of equations can be written as follows:

$$
\mathbf{M} \ddot{\boldsymbol{U}}+\boldsymbol{F}^{\mathrm{i}}=\boldsymbol{F}^{\mathrm{e}}
$$

Here, it should be noted that the above equation has the same form as that obtained using the FEM. However, the global mass matrix, $\mathbf{M}$, and the internal and external force vectors, $\boldsymbol{F}^{\mathrm{i}}$ and $\boldsymbol{F}^{\mathrm{e}}$, are obtained based on the MK-based shape functions. Also, the global mass matrix can be lumped into a diagonal matrix, $\mathbf{M}_{\mathrm{L}}$, to reduce computational costs. By using the explicit central difference method, Eq. (49) can be incrementally solved by using the following explicit equations:

$$
\begin{gathered}
\ddot{\boldsymbol{U}}^{(n)}=\mathbf{M}_{\mathrm{L}}^{-1}\left(\boldsymbol{F}^{\mathrm{e}(n)}-\boldsymbol{F}^{\mathbf{i}(n)}\right) \\
\dot{\boldsymbol{U}}^{\left(n+\frac{1}{2}\right)}=\dot{\boldsymbol{U}}^{\left(n-\frac{1}{2}\right)}+\frac{\Delta t^{(n+1)}+\Delta t^{(n)}}{2} \ddot{\boldsymbol{U}}^{(n)} \\
\boldsymbol{U}^{(n+1)}=\boldsymbol{U}^{(n)}+\Delta t^{(n+1)} \dot{\boldsymbol{U}}^{\left(n+\frac{1}{2}\right)}
\end{gathered}
$$

\subsection{Implementation of PBCs}

The equivalence approach is a one-step method as it formulates generic equations, based on which the boundary conditions of either a full-size UC or a reduced UC can be calculated. This approach was initially proposed by Whitcomb, Chapman and Tang (2000) based on the concept of equivalent coordinate system (ECS), which was applied to establish relations between the geometry and mechanics quantities (i.e. coordinate, displacement, stress and strain) of two adjacent subdomains and thus to derive the boundary condition for the UC of homogenisation. This approach was later generalised by Tang and Whitcomb (2003) and De Carvalho and Pinho (2015). In this present work, the generalised equivalence approach 9 De Carvalho and Pinho 2015) was adapted to derive the boundary conditions of the normal and off-axis UCs. In such an approach, the boundary condition of a reduced UC is derived based 
on the physical equivalence, the loading equivalence, the admissibility condition between the UC domain and its surrounding subdomains. The generic form of a PBC obtained using the equivalence approach is written as follows, if a time variable $t$ is added:

$$
\boldsymbol{u}(\mathrm{A}, t)-\gamma \mathbf{T} \boldsymbol{u}(\overline{\mathrm{A}}, t)=-\langle\boldsymbol{\varepsilon}(t)\rangle \mathbf{T} \boldsymbol{x}^{\mathrm{O}_{\bar{D}}}
$$

where $\mathrm{A}$ is a point on the boundary of the UC under homogenisation, $\overline{\mathrm{A}}$ is located on the boundary of the equivalent subdomain, $\bar{D}$, and is the mapping point of A, $\gamma$ refers to the load reversal factor and has to be 1 or $-1, \mathbf{T}$ is the transformation matrix between the UC domain and its equivalent subdomain, $\langle\boldsymbol{\varepsilon}(t)\rangle$ stands for the macroscopic strain applied, and $x^{\mathrm{O}_{\bar{D}}}$ denotes the vector of the origin of the equivalent subdomain in the reference system of the UC domain. By using Eq. (53) and considering the geometry relations between the UC domain and the surrounding subdomains, as shown in Fig. 5, the boundary conditions for the normal and off-axis UCs of plain woven composites under each admissible loading case can be derived. For simplicity, the detailed boundary conditions are not listed.

To solve Eqs. (50) (52) under the constraint of the time-dependent PBC, i.e. Eq. (53), all field nodes used to discretise the UC under homogenisation are re-ordered into three subsets, i.e. the internal nodes, $\boldsymbol{x}_{I i}\left(I=1, \ldots, n_{i}\right)$, the nodes on the negative boundary, $\boldsymbol{x}_{J-}\left(J=1, \ldots, n_{-}\right)$, and those on the positive boundary, $\boldsymbol{x}_{K+}\left(K=1, \ldots, n_{+}\right)$. By numbering these node sets sequentially, the global displacement vector can be reorganised such that it consists of $\boldsymbol{U}_{i}, \boldsymbol{U}_{-}$, and $\boldsymbol{U}_{+}$, which collect the displacements of the internal nodes, the field nodes on the negative boundary and those on the positive boundary, as follows:

$$
\boldsymbol{U}=\left\{\begin{array}{lll}
\boldsymbol{U}_{i} & \boldsymbol{U}_{-} & \boldsymbol{U}_{+}
\end{array}\right\}^{\mathrm{T}}
$$

Similarly, the global mass matrix and force vectors defined in Eqs. (50) (52) can be reorganised, leading to the following equations:

$$
\left\{\begin{array}{l}
\ddot{\boldsymbol{U}}_{i}^{(n)} \\
\ddot{\boldsymbol{U}}_{-}^{(n)} \\
\ddot{\boldsymbol{U}}_{+}^{(n)}
\end{array}\right\}=\left[\begin{array}{ccc}
\left(\mathbf{M}_{\mathrm{L}}\right)_{i i}^{-1} & \mathbf{0} & \mathbf{0} \\
\mathbf{0} & \left(\mathbf{M}_{\mathrm{L}}\right)_{--}^{-1} & \mathbf{0} \\
\mathbf{0} & \mathbf{0} & \left(\mathbf{M}_{\mathrm{L}}\right)_{++}^{-1}
\end{array}\right]\left(\left\{\begin{array}{c}
\boldsymbol{F}_{i}^{\mathrm{e}(n)} \\
\boldsymbol{F}_{-}^{\mathrm{e}(n)} \\
\boldsymbol{F}_{+}^{\mathrm{e}(n)}
\end{array}\right\}-\left\{\begin{array}{c}
\boldsymbol{F}_{i}^{\mathrm{i}(n)} \\
\boldsymbol{F}_{-}^{\mathrm{i}(n)} \\
\boldsymbol{F}_{+}^{\mathrm{i}(n)}
\end{array}\right\}\right)
$$




$$
\begin{gathered}
\left\{\begin{array}{l}
\dot{\boldsymbol{U}}_{i}^{\left(n+\frac{1}{2}\right)} \\
\dot{\boldsymbol{U}}_{-}^{\left(n+\frac{1}{2}\right)} \\
\dot{\boldsymbol{U}}_{+}^{\left(n+\frac{1}{2}\right)}
\end{array}\right\}=\left\{\begin{array}{l}
\dot{\boldsymbol{U}}_{i}^{\left(n-\frac{1}{2}\right)} \\
\dot{\boldsymbol{U}}_{-}^{\left(n-\frac{1}{2}\right)} \\
\dot{\boldsymbol{U}}_{+}^{\left(n-\frac{1}{2}\right)}
\end{array}\right\}+\frac{\Delta t^{(n)}+\Delta t}{2}\left\{\begin{array}{l}
\ddot{\boldsymbol{U}}_{i}^{(n+1)} \\
\ddot{\boldsymbol{U}}_{-}^{(n)} \\
\ddot{\boldsymbol{U}}_{+}^{(n)}
\end{array}\right\} \\
\left\{\begin{array}{l}
\boldsymbol{U}_{i}^{(n+1)} \\
\boldsymbol{U}_{-}^{(n+1)} \\
\boldsymbol{U}_{+}^{(n+1)}
\end{array}\right\}=\left\{\begin{array}{l}
\boldsymbol{U}_{i}^{(n)} \\
\boldsymbol{U}_{-}^{(n)} \\
\boldsymbol{U}_{+}^{(n)}
\end{array}\right\}+\Delta t^{(n+1)}\left\{\begin{array}{c}
\dot{\boldsymbol{U}}_{i}^{\left(n+\frac{1}{2}\right)} \\
\dot{\boldsymbol{U}}_{-}^{\left(n+\frac{1}{2}\right)} \\
\dot{\boldsymbol{U}}_{+}^{\left(n+\frac{1}{2}\right)}
\end{array}\right\}
\end{gathered}
$$

Base on Eqs. (53) and (54), we can obtain:

$$
\begin{gathered}
\boldsymbol{U}_{+}^{(n)}=\widetilde{\mathbf{T}} \boldsymbol{U}_{-}^{(n)}+\boldsymbol{\xi}^{(n)} \\
\boldsymbol{U}_{+}^{(n+1)}=\widetilde{\mathbf{T}} \boldsymbol{U}_{-}^{(n+1)}+\boldsymbol{\xi}^{(n+1)}
\end{gathered}
$$

where $\widetilde{\mathbf{T}}$ is a matrix collecting the value of $\gamma \mathbf{T}$ associated with each pair of equivalent nodes, and $\boldsymbol{\xi}^{(n)}$ and $\boldsymbol{\xi}^{(n+1)}$ denote the differences between $\boldsymbol{U}_{+}$and $\widetilde{\mathbf{T}} \boldsymbol{U}_{-}$at time steps $n$ and $n+1$, respectively. Substituting the above two equations into Eq. (57) yields:

$$
\dot{\boldsymbol{U}}_{+}^{\left(n+\frac{1}{2}\right)}=\widetilde{\mathbf{T}} \dot{\boldsymbol{U}}_{-}^{\left(n+\frac{1}{2}\right)}+\dot{\boldsymbol{\xi}}^{\left(n+\frac{1}{2}\right)}
$$

where

$$
\dot{\boldsymbol{\xi}}^{\left(n+\frac{1}{2}\right)}=\frac{\left(\xi^{(n+1)}-\xi^{(n)}\right)}{\Delta t^{(n+1)}}
$$

Replacing $n$ in Eq. (60) with $n-1$ produces:

$$
\dot{\boldsymbol{U}}_{+}^{\left(n-\frac{1}{2}\right)}=\widetilde{\mathbf{T}} \dot{\boldsymbol{U}}_{-}^{\left(n-\frac{1}{2}\right)}+\dot{\boldsymbol{\xi}}^{\left(n-\frac{1}{2}\right)}
$$

where

$$
\dot{\xi}^{\left(n-\frac{1}{2}\right)}=\frac{\left(\xi^{(n)}-\xi^{(n-1)}\right)}{\Delta t^{(n)}}
$$

By substituting Eqs. (60) and (62) into Eq. (56), we can obtain:

$$
\ddot{\boldsymbol{U}}_{+}^{(n)}=\widetilde{\mathbf{T}} \ddot{\boldsymbol{U}}_{-}^{(n)}+\ddot{\boldsymbol{\xi}}^{(n)}
$$

where

$$
\ddot{\boldsymbol{\xi}}^{(n)}=\frac{2\left(\dot{\xi}^{\left(n+\frac{1}{2}\right)}-\dot{\xi}^{\left(n-\frac{1}{2}\right)}\right)}{\Delta t^{(n)}+\Delta t^{(n+1)}}
$$


By substituting Eq. (64) into Eq. (55) and performing some mathematical manipulations, the following equation can be obtained:

$$
\widetilde{\ddot{\boldsymbol{U}}}^{(n)}=\widetilde{\mathbf{M}}^{-1}\left(\widetilde{\boldsymbol{F}}^{\mathrm{e}(n)}-\widetilde{\boldsymbol{F}}^{\mathrm{i}(n)}\right)
$$

where

$$
\begin{aligned}
& \widetilde{\tilde{\boldsymbol{U}}}^{(n)}=\left\{\begin{array}{ll}
\ddot{\boldsymbol{U}}_{i}^{(n)} & \ddot{\boldsymbol{U}}_{-}^{(n)}
\end{array}\right\}^{\mathrm{T}} \\
& \widetilde{\mathbf{M}}^{-1}=\left[\begin{array}{cc}
\left(\mathbf{M}_{\mathrm{L}}\right)_{i i} & \mathbf{0} \\
\mathbf{0} & \left(\mathbf{M}_{\mathrm{L}}\right)_{--}+\left(\mathbf{M}_{\mathrm{L}}\right)_{++} \widetilde{\mathbf{T}}
\end{array}\right]^{-1} \\
& \widetilde{\boldsymbol{F}}^{\mathrm{e}(n)}=\left\{\begin{array}{c}
\boldsymbol{F}_{i}^{\mathrm{e}(n)} \\
\boldsymbol{F}_{-}^{\mathrm{e}(n)}+\boldsymbol{F}_{+}^{\mathrm{e}(n)}-\left(\mathbf{M}_{\mathrm{L}}\right)_{++} \ddot{\boldsymbol{\xi}}^{(n)}
\end{array}\right\} \\
& \widetilde{\boldsymbol{F}}^{\mathrm{i}(n)}=\left[\begin{array}{c}
\boldsymbol{F}_{i}^{\mathrm{i}(n)} \\
\boldsymbol{F}_{-}^{\mathrm{i}(n)}+\boldsymbol{F}_{+}^{\mathrm{i}(n)}
\end{array}\right]
\end{aligned}
$$

Since no external force is prescribed when homogenising the response of plain woven composites, the external force components in Eq. (69) vanish, leading to:

$$
\widetilde{\boldsymbol{F}}^{\mathrm{e}(n)}=\left\{\begin{array}{c}
\mathbf{0} \\
-\left(\mathbf{M}_{\mathrm{L}}\right)_{++} \ddot{\boldsymbol{\xi}}^{(n)}
\end{array}\right\}
$$

From Eqs. (56) and (57), we can also obtain:

$$
\begin{gathered}
\widetilde{\boldsymbol{U}}^{\left(n+\frac{1}{2}\right)}=\widetilde{\boldsymbol{U}}^{\left(n-\frac{1}{2}\right)}+\frac{\Delta t^{(n)}+\Delta t^{(n+1)}}{2} \widetilde{\boldsymbol{U}}^{(n)} \\
\widetilde{\boldsymbol{U}}^{(n+1)}=\widetilde{\boldsymbol{U}}^{(n)}+\Delta t^{(n+1)} \widetilde{\boldsymbol{U}}^{\left(n+\frac{1}{2}\right)}
\end{gathered}
$$

where

$$
\begin{gathered}
\widetilde{\boldsymbol{U}}^{\left(n-\frac{1}{2}\right)}=\left\{\begin{array}{ll}
\dot{\boldsymbol{U}}_{i}^{\left(n-\frac{1}{2}\right)} & \dot{\boldsymbol{U}}_{-}^{\left(n-\frac{1}{2}\right)}
\end{array}\right\}^{\mathrm{T}} \\
\widetilde{\boldsymbol{U}}^{(n)}=\left\{\begin{array}{ll}
\boldsymbol{U}_{i}^{(n)} & \boldsymbol{U}_{-}^{(n)}
\end{array}\right\}^{\mathrm{T}}
\end{gathered}
$$

Eqs. (66), (72) and (73) form the final equations for enforcing time-dependent PBCs for homogenising the response of plain woven composites. 


\subsection{A meshfree program}

An in-house computer program implementing the UC models, the constitutive models and the explicit meshfree method was developed for predicting the overall response of plain woven composites. This program starts by discretising a domain representing the mUC under homogenisation using field nodes and backgrounds cells, as shown in Fig. 6. Then, the support domains for all integration points are defined, and the meshfree shape functions are computed for subsequent calculation of the mass matrix and other quantities. Based on the computed quantities and the boundary and initial conditions prescribed to the UC domain, the explicit solution procedure described in Section 4.2 is applied to incrementally calculate the accelerations, velocities and displacements of all field nodes, as well as the strains and stresses of all integration points. Here, it should be noted that the manner of calculating the stress of an integration point depends on the relative position of the integration point within the UC domain, which can be determined using the analytical equations presented in Section 2. For material points within the yarns, the material model for the yarn material is used. Otherwise, the viscoplasticity model is applied to compute the strains and stresses of the integration points representing the polymer matrix. Finally, by volume-averaging the microscopic stresses and considering the history of prescribed strains, the overall stress-strain response of the woven composite under homogenisation can be obtained. From Fig. 6, it is evident that there is no need to explicitly build the geometries of constituents by using the meshfree-based approach. Given that the internal architecture of plain woven composites has been reasonably described using the mUC models, it can be argued that the meshfree-based approach allows to simultaneously address the problems in traditional modelling approaches.

\section{Results and discussion}

To validate the meshfree-based approach, two sets of numerical examples were conducted to simulate the EP121-C15-53 plain woven composite under in-plane normal and off-axis 
tensile loading conditions, as detailed in Table 1. The first set of numerical simulations were based on the normal mUC and to predict the response of the composite subjected to tension in the warp direction $\left(0^{\circ}\right)$ at three different strain rates, i.e. $10^{-1} \mathrm{~s}^{-1}, 10^{-3} \mathrm{~s}^{-1}$ and $10^{-5}$ $\mathrm{s}^{-1}$, while the second set was based on the off-axis mUC and to predict the response of the composite subjected to tension in the off-axis direction $\left(45^{\circ}\right)$ at the same three strain rates. To conduct the above simulations, the normal mUC was discretised with $13 \times 13 \times 7$ field nodes and $60 \times 60 \times 30$ background cells. For the off-axis mUC, it was discretised with $10 \times 20 \times 7$ field nodes and $45 \times 90 \times 30$ background cells. The geometrical parameters of this composite material were extracted based on the micrographs shown in Figs. 2(a) 2(b), and the values used were $L=1.0, H=1.0$ and $\lambda=0.9$.

\subsection{Determination of material parameters}

Six samples of uniaxial tensile tests were conducted at six different strain rates to determine the material parameters of the epoxy resin, i.e. EP121. Table 2 shows the testing strain rates and the results of these tests. Here, in calculating $x$ and $y$ using Eqs. (17) and (18), $D_{0}$ was chosen to be $10^{4} \mathrm{~s}^{-1}$, and $\Omega_{\mathrm{m}}$ was estimated to be $68 \%$ of the maximum saturation stress. Based on the data given in Table $2, n_{\mathrm{r}}$ and $Z_{0}$ were computed following the least-squares regression analysis shown in Fig. 7(a). In terms of $q$, six different values were calculated using Eq. (15), and the average value was used. It should be noted that the test data in Table 2 were also employed to determine the elastic modulus, Poisson's ratio and the ratedependence parameter, $C_{\mathrm{m} \varepsilon}$. Here, another least-squares regression analysis was performed to determine the value of $C_{\mathrm{m} \varepsilon}$, as shown in Fig. 7(b). The material parameters of the epoxy resin are summarised in Table 3.

UD composite specimens having the same constituents and FVF as the yarn material were used to conduct tensile, compressive and shear tests to determine the elastic moduli and strength properties of the yarn material. In the tensile and compressive tests, the specimens were loaded to complete failure. Then, the stress-strain data in the post-failure regime were collected to perform the least-squares procedure described in Section 3.3.3 to tune the 
damage parameters defined in Eq. (33). The material parameters of the yarn material are also summarised in Table 3.

\subsection{Experimental tests for validation}

To experimentally validate the meshfree based modelling approach, composite panels based on 8 layers of EP121-C15-53 prepreg were fabricated using a hot-press machine. The panels were cured with a dwell temperature of $135{ }^{\circ} \mathrm{C}$ for approximately 70 mins and a pressure of 2 bars. Two sets of experimental tests were conducted to validate the numerical simulations detailed in Table 1. Here, the first set was comprised of three uniaxial tensile tests on $0^{\circ}$ specimens at strain rates of approximately $10^{-1} \mathrm{~s}^{-1}, 10^{-3} \mathrm{~s}^{-1}$ and $10^{-5} \mathrm{~s}^{-1}$. In terms of the second set, it was designed to obtain the overall response of the composite subjected to the same three strain rates in the off-axis direction, i.e. $45^{\circ}$ to the warp direction.

\subsection{Comparison between the meshfree prediction and experimental results}

Fig. 8 compares the predicted and measured stress-strain curves of the plain woven composite under tensile loadings in the warp $\left(0^{\circ}\right)$ direction. It is evident that the stressstrain curves predicted by using the meshfree approach are generally in good agreements with those measured from the experimental tests, with the stress showing a linear increase with the strain, before reaching a maximum point. After this point, the stress instantly drops to zero due to brittle fibre fracture. It is interesting to note that there is no significant difference among the measured stress-strain curves, suggesting that the overall response of the plain woven composite in the warp direction is strain-rate insensitive. By comparing the predicted stress-strain curves, it is confirmed that the rate-independent behaviour of this woven composite in the warp direction has been effectively captured by using the meshfreebased model.

Fig. 9 shows the predicted and tested stress-strain curves of the plain woven composite loaded with the three strain rates in the off-axis $\left(45^{\circ}\right)$ direction. Again, the stress-strain curves predicted using the meshfree-based approach agree reasonably well with the experimental results. Indeed, both the rate-dependent behaviour and nonlinear response in 
the off-axis direction have been successfully captured. In addition, the characteristics of increased maximum stress and decreased failure strain with strain rate have been predicted. It is worth highlighting that such rate-dependent features are similar to those observed in the tests conducted for determining the material properties of the polymer matrix. Therefore, it can be argued that the material behaviour of this woven composite in the off-axis direction is primarily associated with that of the polymer matrix.

Apart from the above findings, the failure strains of this composite, either predicted using the meshfree-based model or measured from the experimental tests, are in the range of approximately $22 \%-25 \%$, which is much larger than those of the polymer matrix $(\sim 7 \%)$ and carbon fibres $(\sim 1.7 \%)$. Apparently, such a large degree of deformation in the off-axis direction was due to the reorientation of the warp and weft yarns, as evidenced in Fig. 10. The good agreements between the predicted and measured failure strains justify the shearmodulus discount approach described in Section 3.3.4.

\section{Conclusions}

Experimental observations show that to capture the physics of the damage mechanisms of the woven composite, the wave architecture and internal geometry needs to be considered explicitly. The application of a systematic framework for determining reduced unit cells and respective periodic boundary conditions had been illustrated. An explicit meshfreebased UC modelling approach that incorporates time-dependent PBCs has been formulated to predict the overall response of plain woven composites. It was argued that the proposed approach eliminates the problems inherently existing in traditional UC modelling approaches by means of implicitly implementing reasonably-described UC models. By comparing the predicted and experimental results, it was demonstrated that both the elastic, rate-insensitive behaviour in the warp direction and the nonlinear, rate-dependent response in the off-axis direction were successfully captured. Consequently, the major contribution of the present research can be interpreted as the development of an UC modelling approach for 
homogenising plain woven composites, as well as an extension of the capability of the existing UC modelling methodologies from predicting the static, elastic properties to the dynamic, nonlinear response of plain woven composites.

\section{References}

Aliabadi, M. H. (2015). Woven Composites (Computational and Experimental Methods in Structures). London, Imperial College Press.

Aminjikarai, S. B., Tabiei, A. (2007). A strain-rate dependent 3-D micromechanical model for finite element simulations of plain weave composite structures. Composite Structures, 81(3), 407-418.

Bacarreza, O., Abe, D., Aliabadi, M.H., Ragavan, N. K. (2012). Micromechanical modeling of advanced composites. Journal of Multiscale Modelling, 4(2), 2-20.

Belytschko, T., Lu, Y. Y., Gu, L. (1994). Element-free Galerkin methods. International Journal for Numerical Methods in Engineering, 37(2), 229-256.

Bogdanovich, A. E. (2006). Multi-scale modeling, stress and failure analyses of 3-D woven composites. Journal of Materials Science, 41(20), 6547-6590.

De Carvalho, N. V., Pinho, (2015). Mechanical Responses and Failure of 2D Woven Composite under Compression, Chapter 2, Woven Composite, Imperial College Press, pp 75-107.

Durville, D. (2010). Simulation of the mechanical behaviour of woven fabrics at the scale of fibers. International Journal of Material Forming, 3(2), 1241-1251.

Hallett, S. R. Green, S.D., B.S. El Said (2015). Modelling 3D Woven Composite Preform Deformation, Chapter, Woven Composite, Imperial College Press, pp14-178

Fish, J., Shek, K. (2000). Multiscale analysis of composite materials and structures. Composites Science and Technology, 60(12-13), 2547-2556.

Goldberg, R. K., Stouffer, D. C. (1998). High strain rate deformation modeling of a polymer matrix composite: Part I - Matrix constitutive equations. Retrieved from https://ntrs.nasa.gov/archive/nasa/casi.ntrs.nasa.gov/19980227139.pdf

Gu, L. (2002). Moving kriging interpolation and element-free Galerkin method. International Journal for Numerical Methods in Engineering, 56(1), 1-11.

Gurit. (2019). Datasheet/EP121 - Epoxy prepreg co-curable with phenolics. Retrieved from https://www.gurit.com/-/media/Gurit/Datasheets/ep-121.pdf

Hashin, Z. (1980). Failure criteria for unidirectional fiber composites. Journal of Applied Mechanics, 47(2), 329-334.

Hill, R. (1965). A self-consistent mechanics of composite materials. Journal of the Mechanics and Physics of Solids, 13(4), 213-222. 
Ishikawa, T., Chou, T. W. (1982). Elastic behavior of woven hybrid composites. Journal of Composite Materials, 16(1), 2-19.

Kress, G. (2012). Examination of Hashin's failure criteria for the second world-wide failure exercise. Journal of Composite Materials, 46(19-20), 2539-2561.

Li, L. Y., Wen, P. H., Aliabadi, M. H. (2011). Meshfree modeling and homogenization of 3D orthogonal woven composites. Composites Science and Technology, 71(15), 17771788 .

Lomov, S. V., Gusakov, A. V., Huysmans, G., Prodromou, A., Verpoest, I. (2000). Textile geometry preprocessor for meso-mechanical models of woven composites. Composites Science and Technology, 60(11), 2083-2095.

Lomov, S. V., Verpoest, I. (2000). Integrated model of textile composite reinforcements. In C. A. Brebbia, W. R. Blain, W. P. DE Wilde (Eds.), Advances in Composite Materials and Structures VII (pp. 367-376). WIT Press.

Shembekar, P. S., Naik, N. K. (1992). Elastic behavior of woven fabric composites: II Laminate analysis. Journal of Composite Materials: 26(15), 2226-2246.

Tang, X. D., Whitcomb, J. D. (2003). General techniques for exploiting periodicity and symmetries in micromechanics analysis of textile composites. Journal of Composite Materials, 37(13), 1167-1189.

University of Nottingham. (2019). TexGen. Retrieved from http://texgen.sourceforge.net

Weeks, C. A., Sun, C. T. (1998). Modelling non-linear rate-dependent behaviour in fibrereinforced composites. Composites Science and Technology, 58(3-4), 603-611.

Weibull, W. (1939). A Statistical Theory of the Strength of Materials. Stockholm, Generalstabens Litografiska Anstalts Förlag.

Wen, P. H., Aliabadi, M. H. (2009). Mesh-free micromechanical model for woven fabric composite elastic moduli. Journal of Multiscale Modelling, 1(2), 303-319.

Wentorf, R., Collar, R., Shephard, M. S., Fish, J. (1999). Automated modeling for complex woven mesostructures. Computer Methods in Applied Mechanics and Engineering, 172(1-4), 273-291.

Whitcomb, J. D., Chapman, C. D., Tang, X. D. (2000). Derivation of boundary conditions for micromechanics analyses of plain and satin weave composites. Journal of Composite Materials, 34(9), 724-747.

Whitcomb, J. D., Tang, X. D. (2001). Effective moduli of woven composites. Journal of Composite Materials, 35(23), 2127-2144.

Tabiei, A., Ivanov, I. (2002). Computational micro-mechanical model of flexible woven fabric for finite element impact simulation. International Journal for Numerical Methods in Engineering, 53(6), 1259-1276.

Tabiei, A., Ivanov, I. (2007). Micro-mechanical model with strain-rate dependency and damage for impact simulation of woven fabric composites. Mechanics of Advanced Materials and Structures, 14(5), 365-377. 
Xu, Z. W., Yang, F. J., Guan, Z. W., Cantwell, W. J. (2016). An experimental and numerical study on scaling effects in the low velocity impact response of CFRP laminates. Composite Structures, 154, 69-78. 


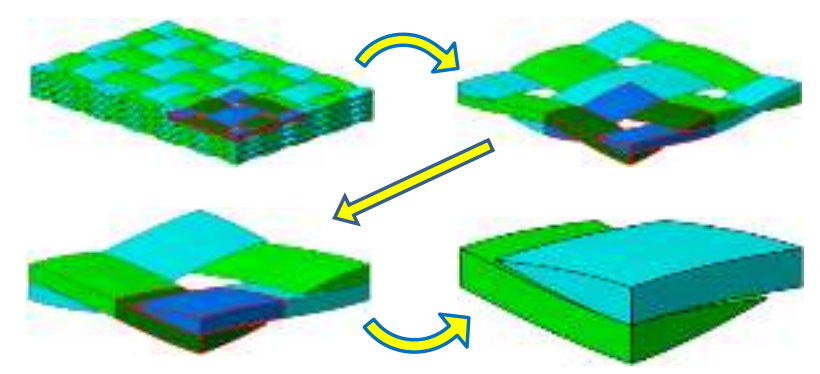

(a) Domain reduction

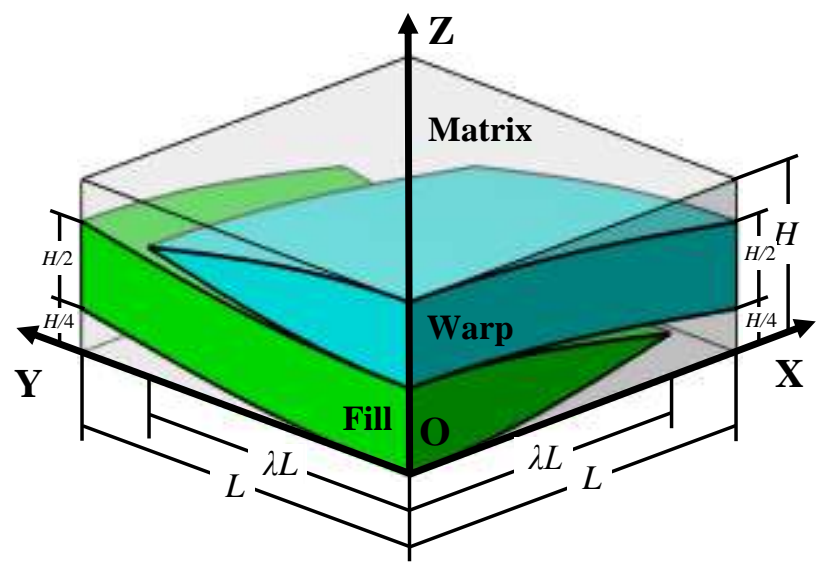

(b) Normal mUC model

Fig. 1: Domain reduction and normal mUC of plain woven composites

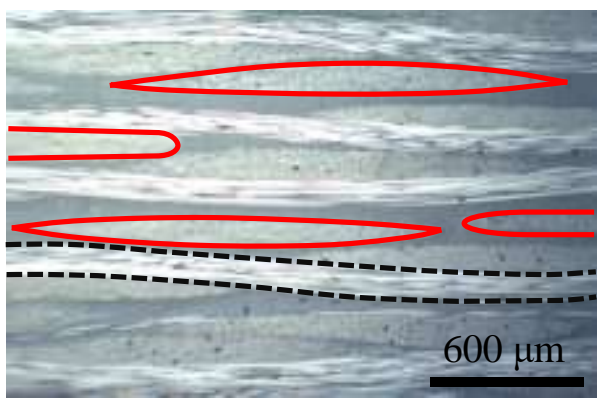

(a) Micrograph at x25 magnification

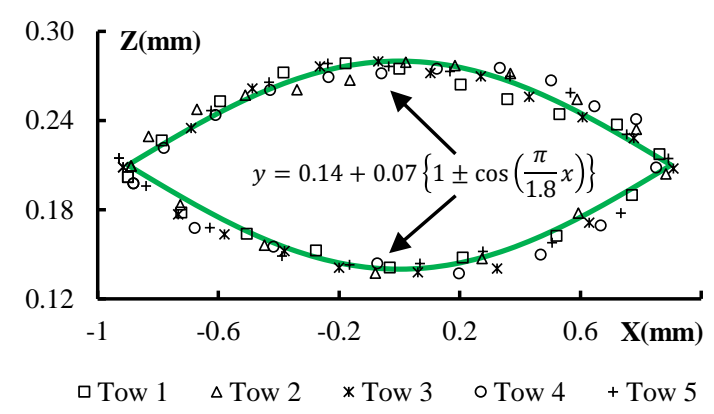

(c) Curve fitting for cross-section

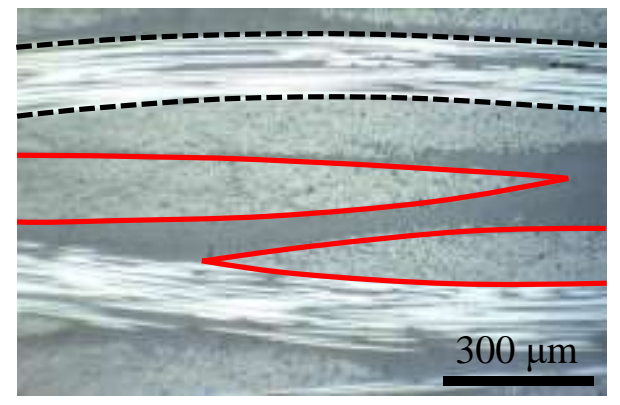

(b) Micrograph at x100 magnification

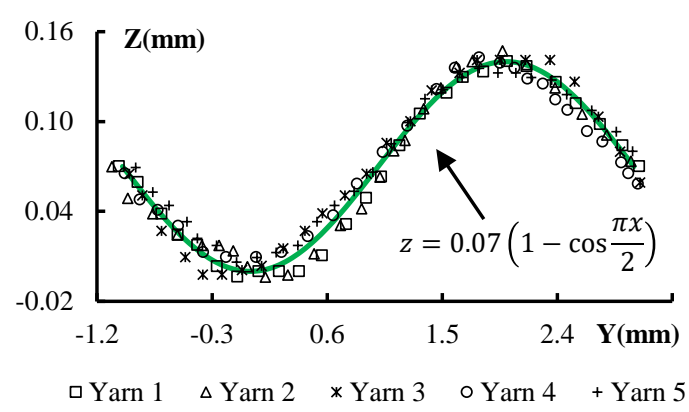

(d) Curve fitting for yarn waviness

Fig. 2: Micrographs and curve fittings for the internal features of EP121-C20-53 


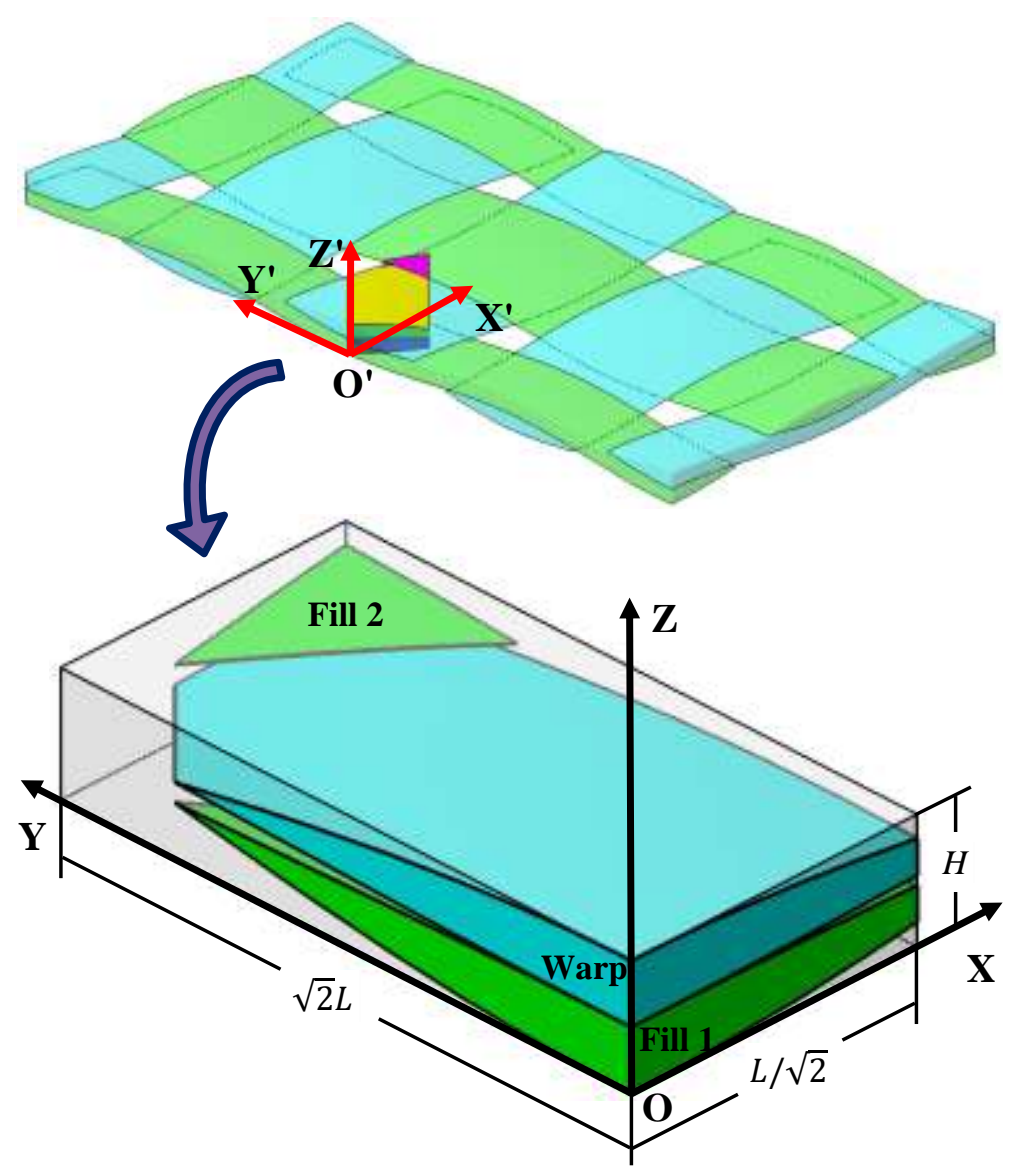

Fig. 3: Off-axis mUC model of plain woven composites

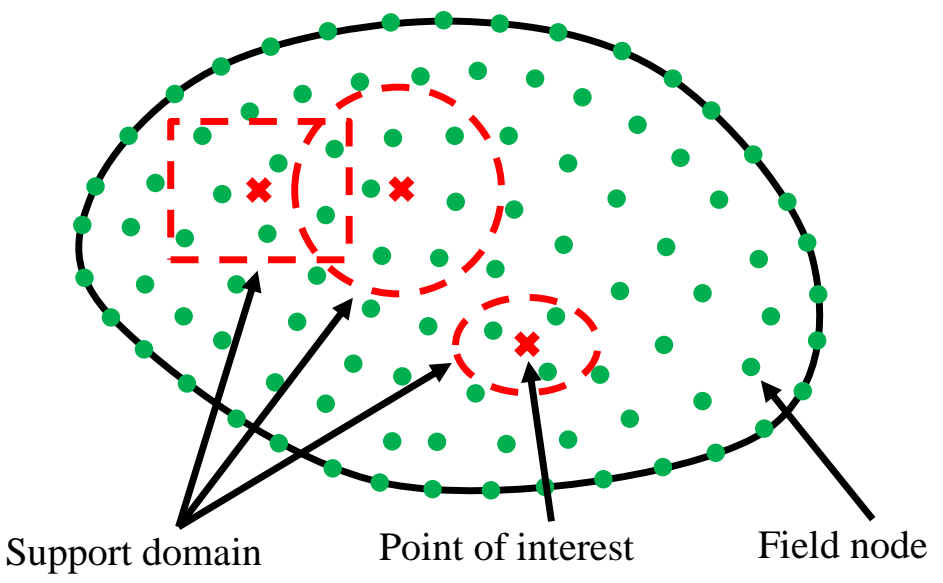

Fig. 4: Node-based discretisation in meshfree methods 


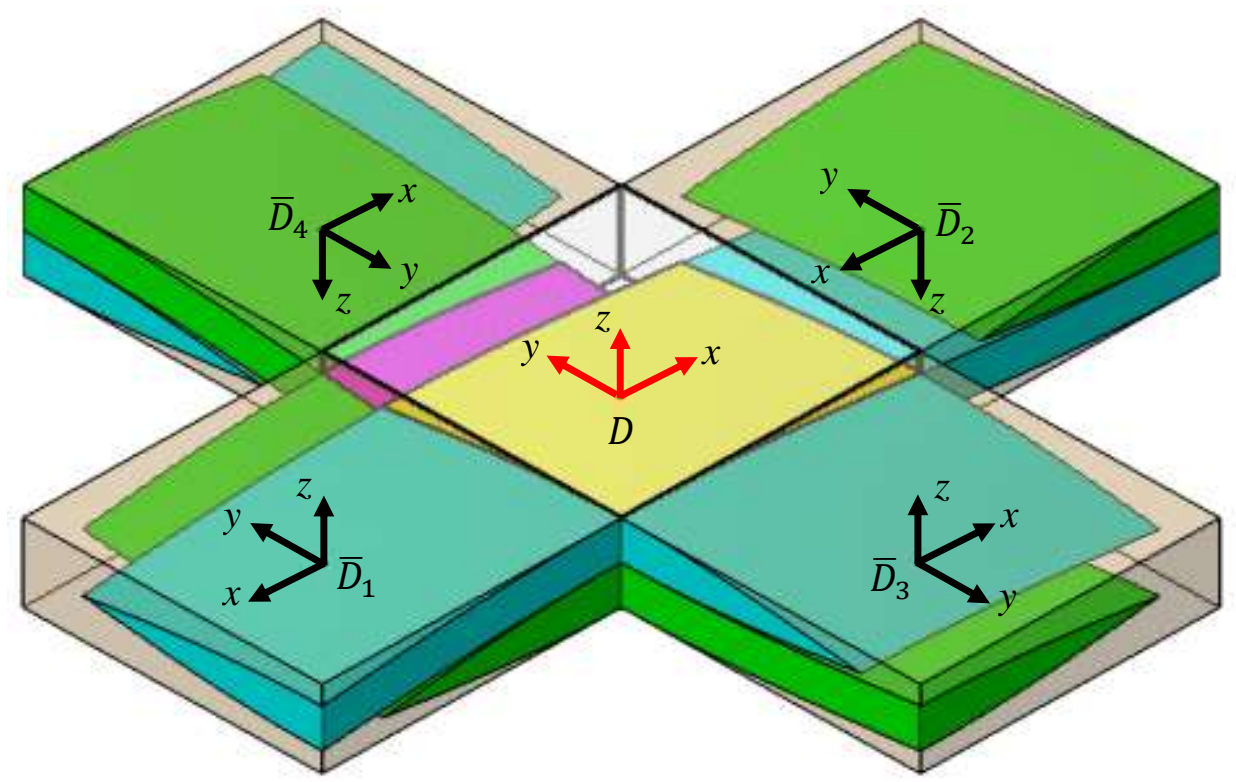

(a) Normal mUC \& its equivalent, adjacent subdomains

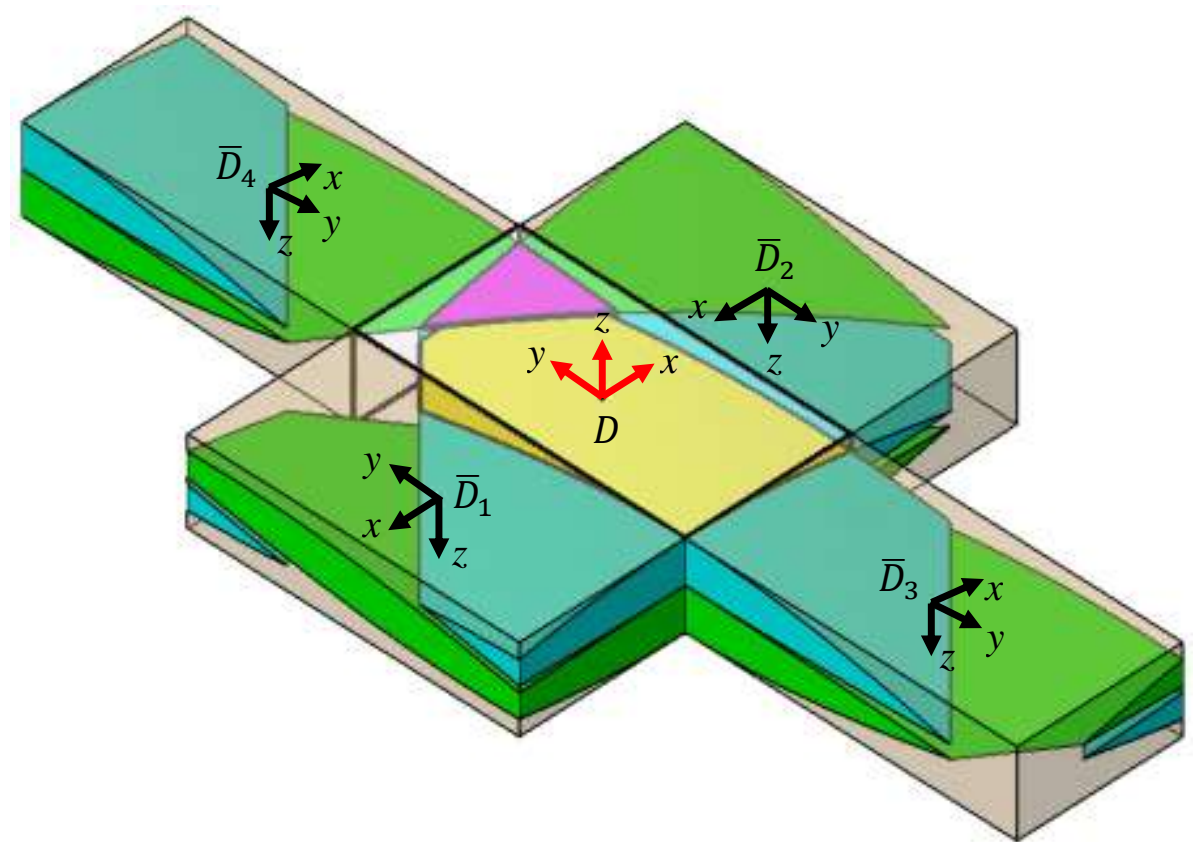

(b) Off-axis mUC \& its equivalent, adjacent subdomains

Fig. 5: Identification of the mUCs and their adjacent subdomains 


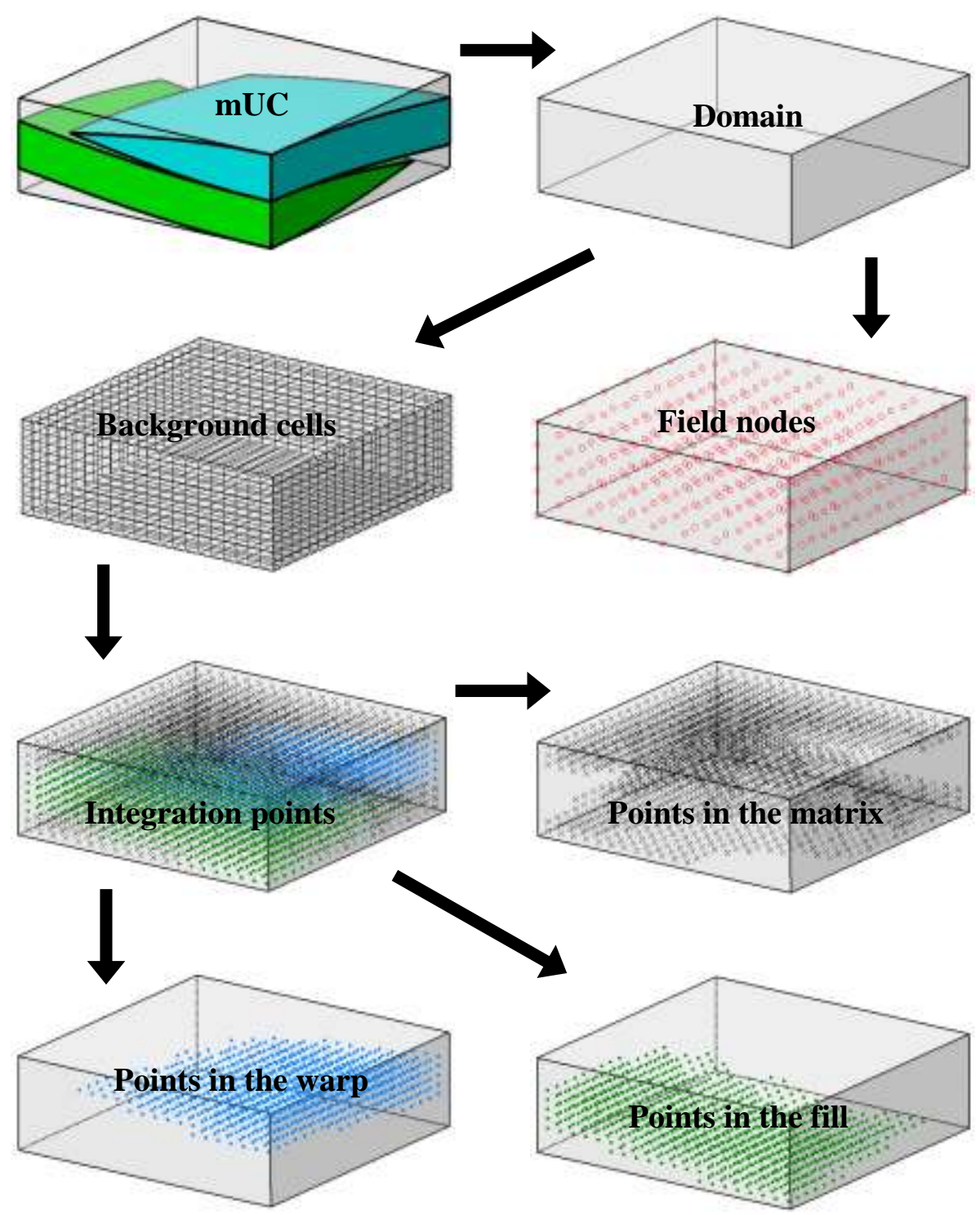

Fig. 6: Meshfree discretisation of the domain representing the mUC

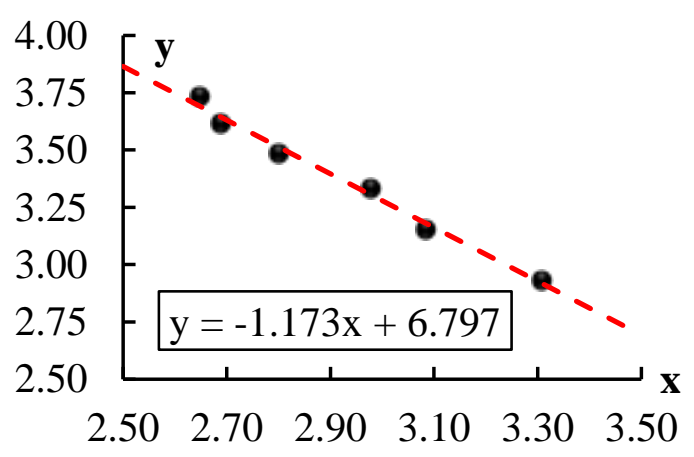

(a) $n_{\mathrm{r}}$ and $Z_{0}$

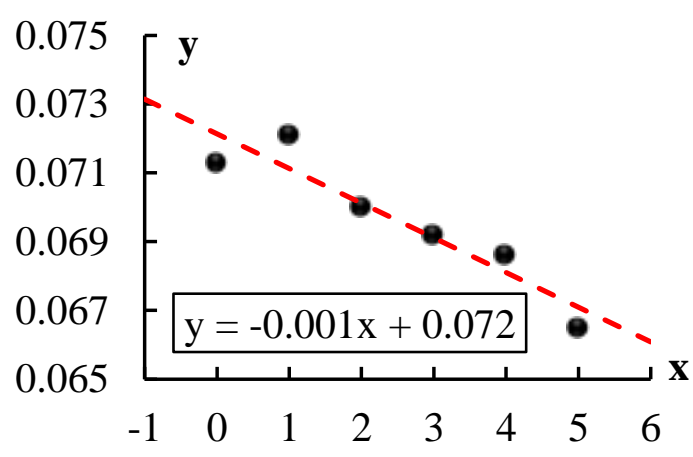

(b) $C_{\mathrm{m} \varepsilon}$

Fig. 7: Least-squares regression analyses for determining the parameters of EP121 


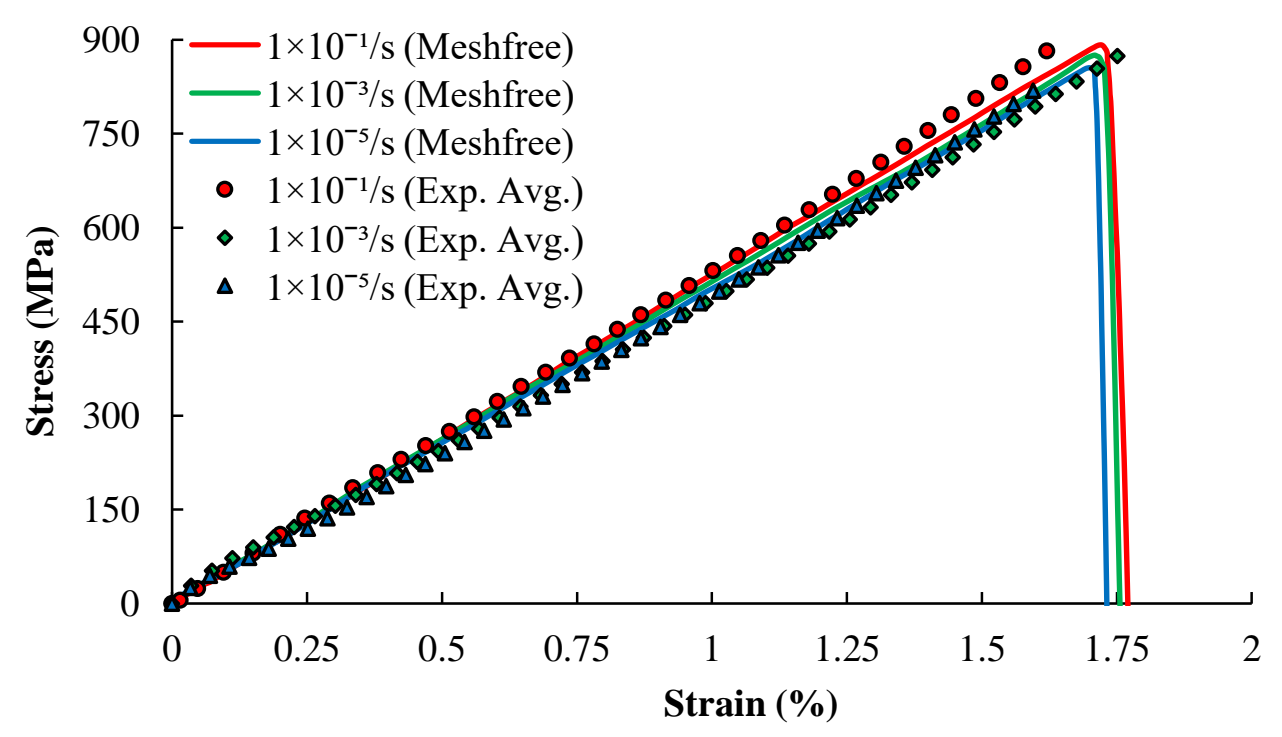

Fig. 8: Meshfree-based predictions \& experimental results for $0^{\circ}$ tension

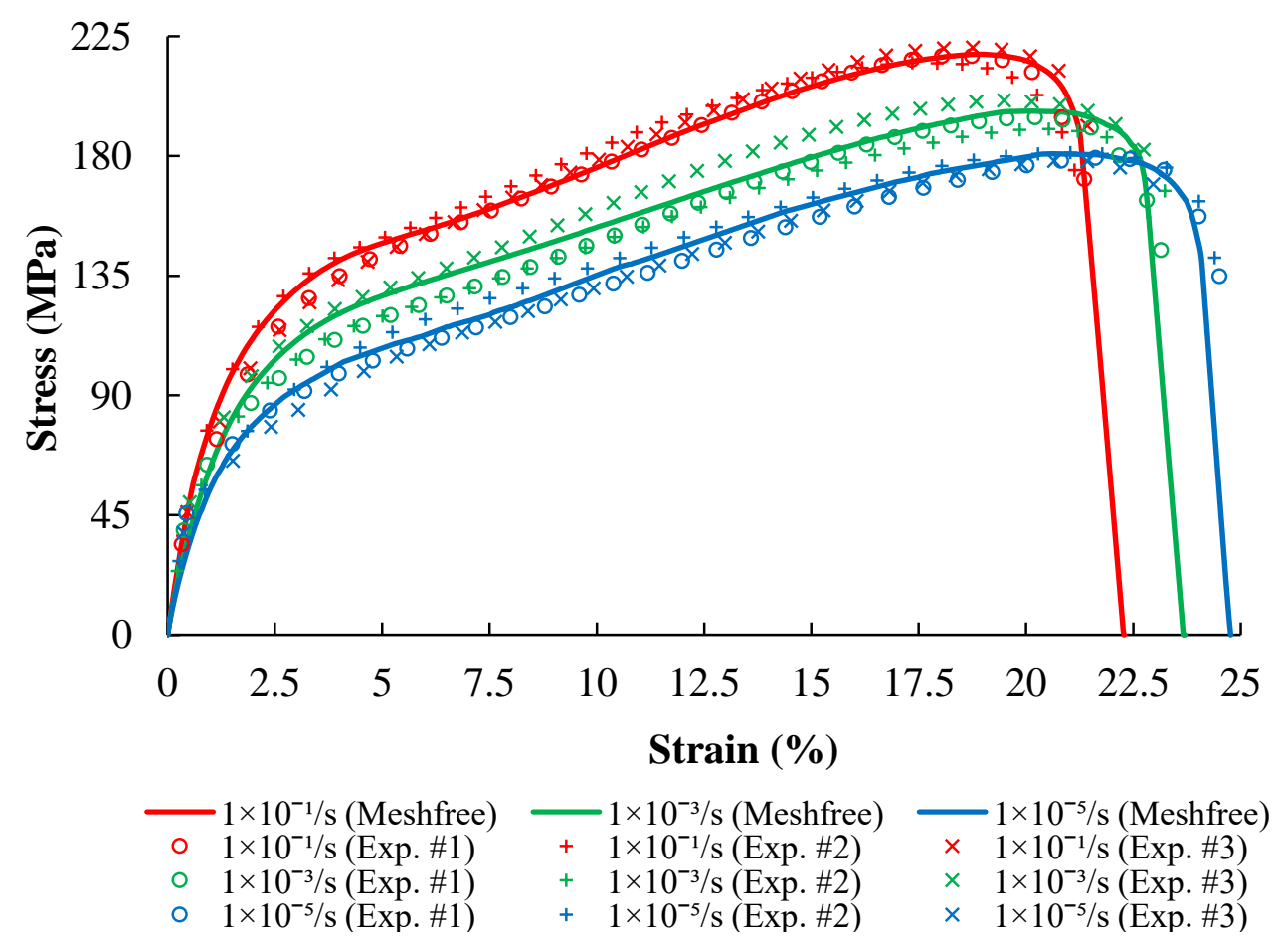

Fig. 9: Meshfree-based predictions \& experimental results for $45^{\circ}$ tension

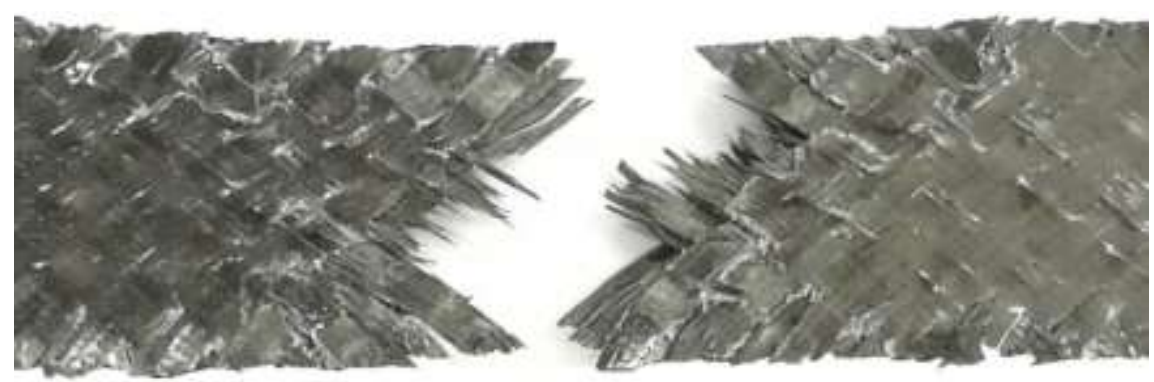

Fig. 10: Typical failure appearance of the woven composite under $45^{\circ}$ tension 
Table 1: Details of the two sets of numerical simulations

\begin{tabular}{ccccc}
\hline \multicolumn{2}{c}{ Simulation } & Rate $\left(\mathrm{s}^{-1}\right)$ & Loading direction & mUC \\
\hline \multirow{2}{*}{ Set } & A1 & $10^{-1}$ & Warp $\left(0^{\circ}\right)$ & Normal \\
A & A2 & $10^{-3}$ & Warp $\left(0^{\circ}\right)$ & Normal \\
& A3 & $10^{-5}$ & Warp $\left(0^{\circ}\right)$ & Normal \\
\hline \multirow{2}{*}{ Set } & B1 & $10^{-1}$ & Off-axis $\left(45^{\circ}\right)$ & Off-axis \\
B & B2 & $10^{-3}$ & Off-axis $\left(45^{\circ}\right)$ & Off-axis \\
& B3 & $10^{-5}$ & Off-axis $\left(45^{\circ}\right)$ & Off-axis \\
\hline
\end{tabular}

Table 2: The averaged results of the six uniaxial tensile tests for EP121

\begin{tabular}{ccccccc}
\hline Sample & $\# 1$ & $\# 2$ & $\# 3$ & $\# 4$ & $\# 5$ & $\# 6$ \\
\hline$\dot{\varepsilon}_{0}$ & $10^{-5}$ & $10^{-4}$ & $10^{-3}$ & $10^{-2}$ & $10^{-1}$ & 1 \\
$\sigma_{\mathrm{s}}$ & 72.32 & 72.91 & 74.68 & 77.85 & 80.07 & 85.54 \\
$\varepsilon_{\mathrm{s}}^{\mathrm{I}}$ & $4.56 \%$ & $4.61 \%$ & $4.47 \%$ & $4.42 \%$ & $4.39 \%$ & $4.25 \%$ \\
$\varepsilon_{\mathrm{mf}}$ & $7.13 \%$ & $7.21 \%$ & $7.00 \%$ & $6.92 \%$ & $6.86 \%$ & $6.65 \%$ \\
$x$ & 2.373 & 2.427 & 2.572 & 2.789 & 2.917 & 3.176 \\
$y$ & 3.731 & 3.614 & 3.482 & 3.329 & 3.149 & 2.929 \\
\hline
\end{tabular}

Table 3: Material inputs of EP121-C15-53 (Modulus in GPa, Strength in MPa)

\begin{tabular}{|c|c|c|c|c|c|c|}
\hline \multirow{4}{*}{$\begin{array}{l}\text { EP121 } \\
\text { Matrix }\end{array}$} & $E_{\mathrm{m}}$ & $v_{\mathrm{m}}$ & $\varepsilon_{\mathrm{m} 0}$ & $C_{\mathrm{m} \varepsilon}$ & $\beta_{\mathrm{m}}$ & $\rho_{\mathrm{m}}\left(\mathrm{g} / \mathrm{cm}^{3}\right)$ \\
\hline & 3.11 & 0.36 & $7.13 \%$ & 0.014 & 3.8 & 1.264 \\
\hline & $D_{0}\left(\mathrm{~s}^{-1}\right)$ & $Z_{0}$ & $n_{\mathrm{r}}$ & $q$ & $\Omega_{\mathrm{m}}$ & \\
\hline & 10000 & 330 & 0.586 & 103 & 58 & \\
\hline \multirow{6}{*}{$\begin{array}{l}\text { Fibre } \\
\text { Yarn }\end{array}$} & $E_{\mathrm{L}}$ & $E_{\mathrm{T}}$ & $v_{\mathrm{LT}}$ & $v_{\mathrm{TT}}$ & $G_{\mathrm{LT}}$ & $\rho_{\mathrm{y}}\left(\mathrm{g} / \mathrm{cm}^{3}\right)$ \\
\hline & 161.64 & 10.57 & 0.27 & 0.33 & 5.52 & 1.614 \\
\hline & $X_{\mathrm{T}} / X_{\mathrm{C}}$ & $Y_{\mathrm{T}} / Y_{\mathrm{C}}$ & $Z_{\mathrm{T}} / Z_{\mathrm{C}}$ & $S_{\mathrm{LT}}$ & $S_{\mathrm{TT}}$ & $S_{\mathrm{TL}}$ \\
\hline & $2687 / 1622$ & $58 / 263$ & $58 / 263$ & 95 & 78 & 80 \\
\hline & $\beta_{1}$ & $\beta_{2}$ & $\beta_{3}$ & $\beta_{4}$ & $\beta_{5}$ & $\beta_{6}$ \\
\hline & 9.0 & 7.6 & 4.7 & 2.8 & 4.7 & 2.8 \\
\hline
\end{tabular}

